\title{
Wind Synthesis and Quality Control of Multiple-Doppler-Derived Horizontal Wind Fields
}

\author{
Katja Friedrich and Martin Hagen \\ Institut für Physik der Atmosphäre, DLR, Oberpfaffenhofen, Wessling, Germany
}

(Manuscript received 16 December 2002, in final form 24 June 2003)

\begin{abstract}
Horizontal wind vector fields can be measured in real time by a bistatic Doppler radar network and can be applied directly for hazard warnings and weather surveillance. Most applications, however, especially for meteorological research and operational meteorology, require quality-controlled wind fields. Therefore, a qualitycontrol scheme is developed that includes algorithms to determine the data quality. The algorithms are applied through a decision criterion, and the quality of wind measurement is weighted with values ranging from 1 to 0 . The results of each weighting algorithm are merged to an average quality index field, which represents the confidence of each horizontal wind measurement. This averaged field is available together with the measured horizontal wind vector field for further applications. This idea is applicable for all kinds of spatial wind field measurements and is applied in the paper for horizontal wind fields measured for monostatic dual- and bistatic dual- and/or multiple-Doppler radar measurements. Wind synthesis and quality control of three-dimensional wind fields are presented for two frontal passages with stratiform precipitation and for a convective situation.
\end{abstract}

\section{Introduction}

High-quality and rapidly updated measured wind vector fields are required for most operational applications, especially for regional weather surveillance, for the nowcasting of meteorological phenomena, and for assimilating wind vector fields into numerical weather prediction (NWP) models. Also, the usage of horizontal wind fields is essential for diagnostic research studies [e.g., the Mesoscale Alpine Program 1999, Improvement of Microphysical Parameterization through Observational Verification Experiment (IMPROVE) 2001, Vertical Exchange and Orography (VERTIKATOR) 2002], warning, and nowcasting of severe weather events at airports and around populated areas (e.g., the Integrated Terminal Weather System (ITWS) at the airports in New York, New York; Orlando, Florida; Dallas-Fort Worth, Texas; and Hong Kong, China). Flow phenomena (e.g., downslope flow, flow blocking) and local wind systems can be characterized by deriving rotation, divergence, and wind shear patterns from three-dimensional wind fields.

Doppler radar systems sample Doppler velocity and reflectivity over a horizontal range of up to $250 \mathrm{~km}$, with a spatial resolution of $100 \mathrm{~m}$ and a temporal resolution within minutes. With a traditional monostatic Doppler radar system, only one wind component out of three can be measured. One reason for the preferred usage of three-dimensional wind vector fields is the fast

Corresponding author address: Katja Friedrich, DLR-Institut für Physik der Atmosphäre, Oberpfaffenhofen, 82234 Wessling, Germany.

E-mail: katja.friedrich@dlr.de and easy detection of signatures in the wind field, especially for users with no experience in interpretation of radar Doppler velocities. Various techniques for scientific and operational usage can be applied to retrieve wind vector fields from Doppler velocity and reflectivity measurements. Alternatively, if a region is monitored by several Doppler weather radars (monostatic multipleDoppler radar network), a wind vector can be determined based on a least squares estimation. The same multiple-Doppler analysis can be applied when the wind vector is observed by one monostatic Doppler weather radar system and several nontransmitting passive receivers at remote sites (bistatic multiple-Doppler network). Thus, the demand for automated schemes creating additional fields that represent the confidence of the wind field measurement is high. The objective of this work was to develop a scheme for an automated quality control and an automated analysis of the confidence of each measurement for horizontal wind fields measured by monostatic dual- and bistatic multipleDoppler radar networks.

Algorithm development of an automated qualitycontrol procedure is mainly limited to precipitation data, for example, for the Tropical Rainfall Measuring Mission (TRMM) ground validation radar data or hydrological applications (Einfalt et al. 2000). Some dual-Doppler analysis programs use the geometrically induced standard deviation of the horizontal wind as a quality index, for example, "CEDRIC" software (Mohr et al. 1986). Standard deviations of the horizontal wind, depending on the number of independent velocity measurements, together with a range depen- 
dency, has been used by Ray et al. (1978). Furthermore, investigations on the error estimation in wind fields have been accomplished for different kinds of singleand multiple-Doppler radar measurements (e.g., Doviak et al. 1976; Testud and Chong 1983; Takaya and Nakazato 2002).

Radar measurements can be contaminated by clutter from either normal propagation (permanent clutter) or anomalous propagation of the radiation, biological targets, or chaff. Other acquisition properties like angular velocity of the antenna, the number of averaged pulses, and aliasing effects also influence the quality of the measured data (Alberoni et al. 2002). Furthermore, radar data have to be corrected for the contribution of the fall speeds of hydrometeors to the radial velocity. However, over the years large efforts have been made to detect and filter meteorological targets from contamination and to remove noisy data by threshold setting during signal processing; for example, for different ground clutterfiltering methods see Lee et al. (1995), Hagen (1997), and Seltmann (2000); and for dealiasing algorithms see Yamada and Chong (1999); James et al. (2000); and James and Houze (2001).

Some applications like ground clutter filtering, removal of erroneous data by default threshold settings, or dealiasing of Doppler velocities using the dual-PRF technique can be performed directly in the signal processor. On the other hand, some algorithms can only be applied afterward, because of a limited communication bandwidth (e.g., data transfer within a bistatic Doppler radar network), or because the entire volume scan is necessary for that application, for example, for dealiasing Doppler velocities, the creation of quality index fields, or dual-Doppler analysis using independent Doppler radar data.

Creating a quality-control scheme is a sophisticated task. If the scheme is too strict, too much data, even higher-quality data, will be rejected. If the scheme is not strict enough, errors can pass. The choice for a tight or loose quality-control scheme will depend mainly on the weather conditions and the number of measurements required. Furthermore, quality-control schemes have to handle a wide array of false-echo scenarios. Most schemes are based on the setting of thresholds that are chosen mostly empirically. Fixed thresholds give problems when applied to different weather situations and for different scientific purposes. The balance between the quality and the number of measurements must be found according to the scientific question to be investigated.

In this paper it is shown how different wind vector components, for instance, from monostatic or bistatic Doppler radar measurements, can be combined in to a horizontal wind field (section 3), and how a qualitycontrol scheme can be applied to the wind vector field (section 4). The quality control presented in this paper is a fundamental part of an automated evaluation tool for bistatically measured wind fields, which includes Doppler velocity dealiasing (Friedrich and Caumont
2004) and a three-dimensional wind vector retrieval (Protat and Zawadzki 1999). The quality-control scheme can be divided into three parts. First, the signal quality of each range gate is controlled based on spectral width measurements, and noisy data are removed in order to increase the speed and efficiency of the following algorithms (section 4a). In the second step described in section $4 \mathrm{c}$, the quality of the measurements is analyzed by means of quality index fields with values ranging from 0 to 1 . The quality index fields cover, for instance, the geometrically induced accuracy of the Doppler velocity measurement and the amount of sidelobe contamination. These fields can be merged into an average quality-control field, which is available together with the wind vector field to the user. In the third part, described in sections $4 \mathrm{~b}$ and $4 \mathrm{~d}$, signatures within the wind field are checked according to their temporal and spatial persistence. The application of the quality-control scheme is demonstrated for different weather situations in sections 5 and 6.

The algorithms presented in this paper are a first attempt on creating an automated quality-control scheme that can be applied to all kinds of wind field measurements, for example, bistatic or monostatic multipleDoppler radar data. They can be supplemented or expanded. The quality-control scheme together with the automated evaluation tool can be applied immediately after the volume scan has been completed.

\section{The monostatic and bistatic Doppler radar network}

The bistatic Doppler radar system is an instrument capable of measuring simultaneously several components of the wind vector, which can be combined in to a horizontal wind vector field in real time (Wurman et al. 1993). Several receivers separated spatially from the transmitter are implemented around the monostatic Doppler radar.

The bistatic Doppler radar network at the Deutsches Zentrum für Luft und Raumfahrt (DLR) at Oberpfaffenhofen (OP) near Munich in southern Germany consists of the monostatic polarization diversity Doppler radar (POLDIRAD; Schroth et al. 1988) and three bistatic receivers at remote sites, each containing both at least one antenna and a signal processor. In Fig. 1, the location of the three bistatic receivers at Lichtenau, Lagerlechfeld, and Ried, together with the respective look angles of the bistatic antennas, are illustrated. The investigation area, indicated schematically in Fig. 1, is restricted in azimuthal and vertical directions by the received power pattern of the bistatic antenna, which has a horizontal angular aperture covering about $60^{\circ}$, and in range by the sample spacing.

The receiver system at Lagerlechfeld is equipped with two south-facing antennas, which have a vertical angular aperture covering $1^{\circ}-9^{\circ}$ for measurements within the boundary layer and $1^{\circ}-23^{\circ}$ for measuring within thunderstorms. At Ried and Lichtenau, one antenna, having a 
vertical aperture of $8^{\circ}$, has been used for the examples presented in this paper. At the moment, the bistatic radar network at OP covers an area of about $50 \mathrm{~km} \times 50 \mathrm{~km}$ horizontally with a height up to $5 \mathrm{~km}$ within stratiform precipitation and $14 \mathrm{~km}$ within thunderstorms both at a range of $35 \mathrm{~km}$. Within this area, horizontal wind fields are determined exactly in the dual-Doppler area (in Fig. 1 , hatched) and overdetermined in triple- and/or quadruple-Doppler areas (in Fig. 1, cross hatched).

Generally, investigations on measurement characteristics within a bistatic Doppler radar system and wind vector field analysis had already been initiated, for example, for supercell hailstorms and convective systems, by Skolnik (1990), Satoh and Wurman (1999), de Elia and Zawadzki (2000, 2001), Friedrich et al. (2000), and Takaya and Nakazato (2002).

Horizontal wind fields using monostatic dual-Doppler analysis were determined using POLDIRAD and the monostatic Doppler radar system operated by the $\mathrm{Me}$ teorological Observatory Hohenpeissenberg (hereinafter HP) of the German Weather Service located on top of Mount Hohenpeissenberg (cf. Fig. 1).

\section{Wind synthesis}

To estimate the three components of the wind vector, independent measurements by three Doppler radar systems are necessary. These can be either monostatic or bistatic radar systems. As shown by Wurman et al. (1993), the direct determination of the vertical wind component, $w$, is very inaccurate at low elevations, because the contribution of the vertical component of the wind velocity is small in the measured Doppler velocity for $1^{\circ} \leq \theta_{b} \leq 8^{\circ}$, corresponding to $0.02 w \leq \sin \left(\theta_{b}\right) w$ $\leq 0.14 w$. It seems more appropriate to integrate the vertical velocity from the horizontal wind field. Recent studies using variational analysis (e.g., Protat and Zawadzki 1999; Montmerle et al. 2001; Nissen et al. 2001) show reliable results. Therefore, the direct determination of the wind field is limited to the horizontal wind vector field. In the following case studies, a variational technique is used to derive the $3 \mathrm{D}$ wind field consisting of $u, v$, and $w$ from the integration of an airmass continuity equation developed and adjusted by Laroche and Zawadzki (1994, 1995) and Protat and Zawadzki (1999).

Here, a general version of the horizontal wind vector field determination is presented for all kinds of multipleDoppler velocity processing, that is, dual-Doppler, triple-Doppler, and so on. For horizontal wind field determination, one transmitting source and at least two receivers are necessary. With two measured Doppler velocities, the equation system to calculate the horizontal wind vector field is exactly determined.

In a multiple-Doppler network consisting of $n$ receivers $(n \geq 2), n$ Doppler velocities, $v_{m_{n}}$, are measured. This leads to an overdetermined equation system that can be written as

$$
\left[\begin{array}{cc}
\frac{\sin \left(\phi_{b_{1}}\right) \cos \left(\theta_{b_{1}}\right)+\sin \left(\phi_{t}\right) \cos \left(\theta_{t}\right)}{2 \cos \left(\gamma_{1} / 2\right)} & \frac{\cos \left(\phi_{b_{1}}\right) \cos \left(\theta_{b_{1}}\right)+\cos \left(\phi_{t}\right) \cos \left(\theta_{t}\right)}{2 \cos \left(\gamma_{1} / 2\right)} \\
\frac{\sin \left(\phi_{b_{2}}\right) \cos \left(\theta_{b_{2}}\right)+\sin \left(\phi_{t}\right) \cos \left(\theta_{t}\right)}{2 \cos \left(\gamma_{2} / 2\right)} & \frac{\cos \left(\phi_{b_{2}}\right) \cos \left(\theta_{b_{2}}\right)+\cos \left(\phi_{t}\right) \cos \left(\theta_{t}\right)}{2 \cos \left(\gamma_{2} / 2\right)} \\
\vdots & \frac{\cos \left(\phi_{b_{n}}\right) \cos \left(\theta_{b_{n}}\right)+\cos \left(\phi_{t}\right) \cos \left(\theta_{t}\right)}{2 \cos \left(\gamma_{n} / 2\right)}
\end{array}\right]\left(\begin{array}{c}
u \\
v
\end{array}\right)=\left(\begin{array}{c}
v_{m_{1}} \\
v_{m_{2}} \\
\vdots \\
v_{m_{n}}
\end{array}\right),
$$

with $\phi_{b_{i}}, \theta_{b_{i}}$ being azimuth and elevation angles at the $i$ th receiver. The azimuth and elevation angle of the transmitting radar are $\phi_{t}$ and $\theta_{t}$, respectively. The scattering angle of the respective bistatic Doppler radar system related to the $i$ th bistatic receiver is denoted by $\gamma_{i}$. If the Doppler velocity measurement is achieved by a monostatic Doppler radar, the geometrical parameters change to $\phi_{b}=\phi_{t}, \theta_{b}=\theta_{t}$, and $\gamma$ $=0$, and Eq. (1) becomes the already known equation to determine horizontal wind vectors from measurements with several monostatic radar systems (Ray et al. 1978).

This overdetermined equation system can be solved in a least squares sense. Therefore, the normal equations are solved as

$$
\underbrace{\left(\begin{array}{ll}
a_{11} & a_{12} \\
a_{21} & a_{22}
\end{array}\right)}_{\mathbf{A}}\left(\begin{array}{l}
u \\
v
\end{array}\right)=\left(\begin{array}{l}
b_{1} \\
b_{2}
\end{array}\right),
$$

where

$$
\begin{aligned}
& a_{11}=\sum_{i=1}^{n}[\left.\frac{\sin \left(\phi_{b_{i}}\right) \cos \left(\theta_{b_{i}}\right)+\sin \left(\phi_{t}\right) \cos \left(\theta_{t}\right)}{2 \cos \left(\gamma_{i} / 2\right)}\right]^{2}, \\
& a_{12}=a_{21}=\sum_{i=1}^{n}\left[\frac{\cos \left(\phi_{b_{i}}\right) \cos \left(\theta_{b_{i}}\right)+\cos \left(\phi_{t}\right) \cos \left(\theta_{t}\right)}{2 \cos \left(\gamma_{i} / 2\right)}\right. \\
&\left.\times \frac{\sin \left(\phi_{b_{i}}\right) \cos \left(\theta_{b_{i}}\right)+\sin \left(\phi_{t}\right) \cos \left(\theta_{t}\right)}{2 \cos \left(\gamma_{i} / 2\right)}\right],
\end{aligned}
$$




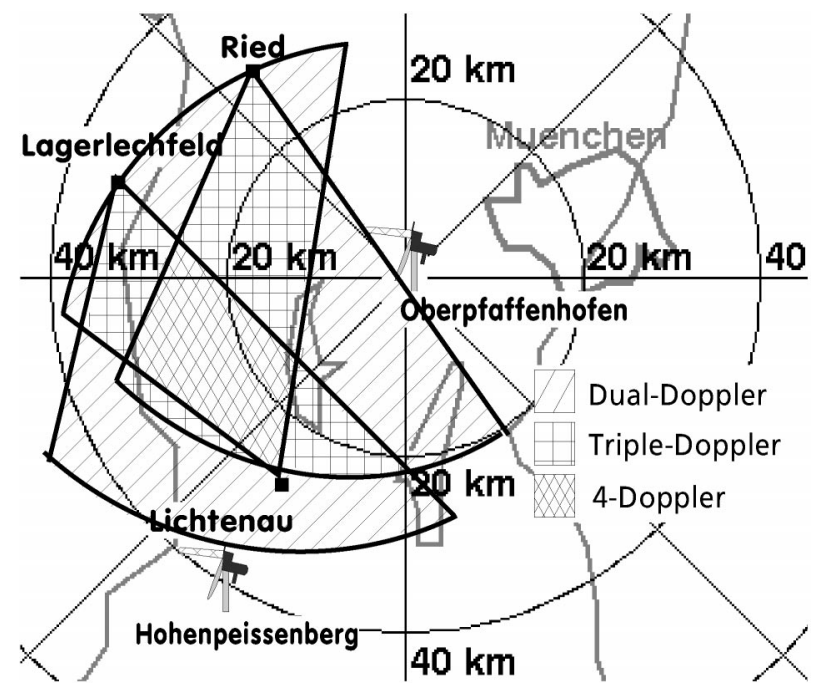

FIG. 1. Map of the bistatic multiple-Doppler radar network at the DLR in OP in southern Germany consisting of POLDIRAD and three bistatic receivers located at Lichtenau, Lagerlechfeld, and Ried. The investigation area is restricted by the horizontal antenna aperture of the bistatic antennas and the range resolution. The equation system to calculate the horizontal wind field is exactly determined in the dual-Doppler areas (hatched) and overdetermined in the triple- or quadruple-Doppler areas (cross hatched). Horizontal wind fields are derived additionally from measurements of POLDIRAD and the radar at Hoherpeissenberg using monostatic dual-Doppler analysis. More explanations are available in the text.

$$
\begin{aligned}
a_{22} & =\sum_{i=1}^{n}\left[\frac{\cos \left(\phi_{b_{i}}\right) \cos \left(\theta_{b_{i}}\right)+\cos \left(\phi_{t}\right) \cos \left(\theta_{t}\right)}{2 \cos \left(\gamma_{i} / 2\right)}\right]^{2}, \\
b_{1} & =\sum_{i=1}^{n} v_{m_{i}} \frac{\sin \left(\phi_{b_{i}}\right) \cos \left(\theta_{b_{i}}\right)+\sin \left(\phi_{t}\right) \cos \left(\theta_{t}\right)}{2 \cos \left(\gamma_{i} / 2\right)}, \text { and } \\
b_{2} & =\sum_{i=1}^{n} v_{m_{i}} \frac{\cos \left(\phi_{b_{i}}\right) \cos \left(\theta_{b_{i}}\right)+\cos \left(\phi_{t}\right) \cos \left(\theta_{t}\right)}{2 \cos \left(\gamma_{i} / 2\right)} .
\end{aligned}
$$

The solution of Eq. (1) is given as

$$
\left(\begin{array}{l}
u \\
v
\end{array}\right)=\frac{1}{\operatorname{det}(\mathbf{A})}\left(\begin{array}{cc}
a_{22} & -a_{12} \\
-a_{12} & a_{11}
\end{array}\right)\left(\begin{array}{l}
b_{1} \\
b_{2}
\end{array}\right),
$$

where

$$
\operatorname{det}(\mathbf{A})=a_{11} a_{22}-a_{12}^{2} .
$$

The horizontal wind field can be calculated with

$$
\begin{aligned}
& u=\frac{1}{\operatorname{det}(\mathbf{A})}\left(a_{22} b_{1}-a_{12} b_{2}\right) \quad \text { and } \\
& v=\frac{1}{\operatorname{det}(\mathbf{A})}\left(a_{11} b_{2}-a_{12} b_{1}\right) .
\end{aligned}
$$

The Doppler velocities can be measured by both monostatic and/or bistatic receivers.

Note that in the monostatic case, two radial velocity components are merged to the wind vectors with an intersection angle ranging between $0^{\circ}$ and $180^{\circ}$. In the bistatic case, the radial velocity component and the com- ponent perpendicular to the ellipse of constant time delay are used (Protat and Zawadzki 1999). Here, the bistatic intersection angle ranges from $0^{\circ}$ to $90^{\circ}$.

\section{The quality-control scheme}

a. The decision criterion based on the signal quality

In the DLR bistatic Doppler radar network, the Doppler power spectrum is obtained at the remote receiver. Only the processed data of power, Doppler velocity, and normalized coherent power $(\mathrm{NCP})^{1}$ can be transfered to the central hub computer located at the transmitting site due to a limited communication bandwidth. At the central hub computer the data of all receivers are collected, and the horizontal wind field is determined and quality controlled.

First data with large velocity dispersion and low reflectivity factors are rejected using an empirically chosen threshold of NCP $\leq 0.3$. As an example of applying the NCP criterion on measured data, Fig. 2 presents a plan position indicator (PPI) at $1^{\circ}$ elevation of the horizontal wind vector field superimposed on the NCP field measured by the receiver at Lichtenau. The horizontal wind was determined using velocity information from the receivers at Lichtenau and OP. In Fig. 2a the NCP criterion is not applied, while in Fig. $2 b$ noisy data are removed using the NCP criterion. As a result, the NCP criterion is applied to remove noisy, spurious data in order to improve the efficiency of the succeeding algorithms, for example, the dealiasing algorithm, and the variational analysis method used to derive $3 \mathrm{D}$ wind fields and to remove artificial gradients that can be misinterpreted.

At monostatic Doppler radars, a signal quality index (SQI) with $\mathrm{SQI}=\left|R_{1}\right| / R_{0}$ is usually applied in signal processors, for example, in Sigmet's signal processor (see RVP7 user's manual, 5-14; available online at ftp://ftp.sigmet.com/outgoing/manuals/rvp7user/).

\section{b. Utilizing data consistency based on an internal check}

By means of an internal consistency check (referred to hereinafter as DCIC), data contamination caused by the sidelobe of the transmitting beam pattern, the migration of birds, and internal hardware problems (e.g., receiver synchronization) can be detected. If data inconsistency is detected at one receiver, data measured by this receiver are completely excluded from further data processing (e.g., variational analysis method, wind synthesis). However, there is a limitation to this control because contamination can only be identified within

\footnotetext{
${ }^{1}$ The index related inversely to the spectral width, ranging from 0 to 1 , is calculated at the bistatic receivers as NCP $=\left|R_{1}\right| / R_{0}$ with $R_{0}$ and $R_{1}$ being the 0 th and 1 st moment of the autocorrelation function taken from the Doppler power spectrum (for more details see Friedrich 2002, p. 114).
} 
(a)

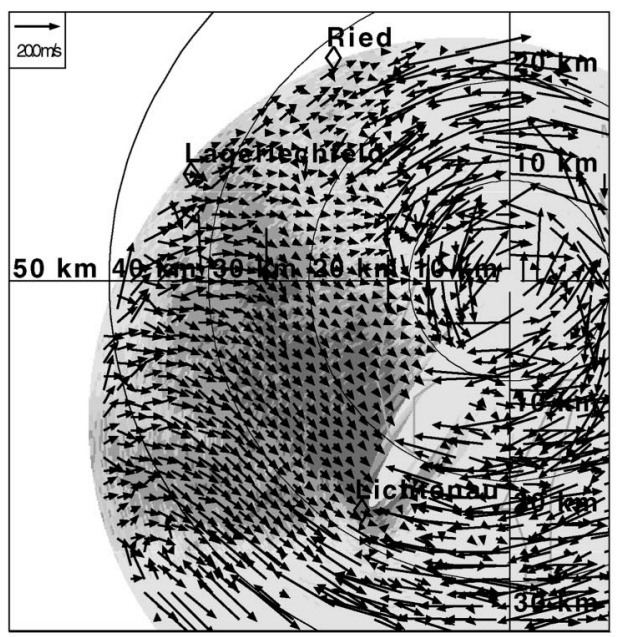

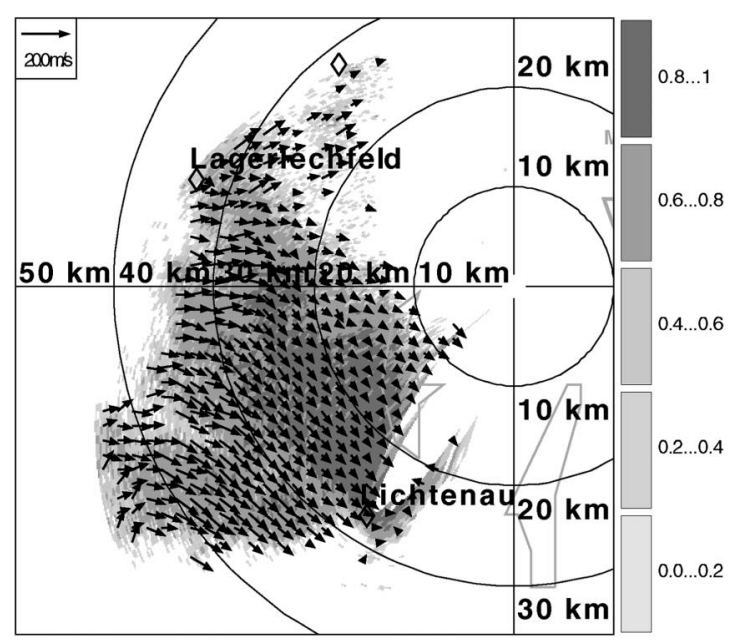

FIG. 2. PPI at $1^{\circ}$ elevation of the horizontal wind $\left(\mathrm{m} \mathrm{s}^{-1}\right.$; arrows) underlaid by the NCP measured by the receiver at Lichtenau at 0945 UTC 6 Jul 2002 (a) without and (b) with applying the NCP criterion on the measured data. The horizontal wind vector is determined by using the Doppler velocity sampled by the receivers at Lichtenau and OP. these areas where the equation system to determine a horizontal wind field is overdetermined.

The idea of the internal check is to determine the horizontal wind field with Doppler velocities measured by two receivers, $i$ and $j$. From this horizontal wind field, the Doppler velocity, which should be measured by the receiver, $k$, not involved in the dual-Doppler analysis, is reconstructed. Within the multiple-Doppler areas, the reconstructed, $v_{e}^{i j}(k)$, and the actual observed Doppler velocities, $v_{e}(k)$, are then compared.

The Doppler velocity measured by the receiver $i$ can be expressed analog to Eq. (1) as

$$
v_{e}(i)=a_{i} u+b_{i} v
$$

with

$$
\begin{aligned}
a_{i} & =\frac{\sin \phi_{b_{i}} \cos \theta_{b_{i}}+\sin \phi_{t} \cos \theta_{t}}{2 \cos (\gamma / 2)} \text { and } \\
b_{i} & =\frac{\cos \phi_{b_{i}} \cos \theta_{b_{i}}+\cos \phi_{t} \cos \theta_{t}}{2 \cos (\gamma / 2)} .
\end{aligned}
$$

In the monostatic case $v_{e}(i)$ becomes $v_{r}$ with $\gamma=0, \phi_{b}$ $=\phi_{t}$, and $\theta_{b}=\theta_{t}$. The horizontal wind vector using two Doppler velocity components, $v_{e}(i)$ and $v_{e}(j)$, measured by receivers $i$ and $j$, can be determined exactly as

$$
\begin{aligned}
& u_{i j}=\frac{b_{j} v_{e}(i)-b_{i} v_{e}(j)}{\Delta_{i j}} \text { and } \\
& v_{i j}=\frac{a_{i} v_{e}(j)-a_{j} v_{e}(i)}{\Delta_{i j}}
\end{aligned}
$$

with $\Delta_{i j}=a_{i} b_{j}-a_{j} b_{i}$. The velocity component of a third receiver, $k$, not involved in the dual-Doppler anal- ysis [Eqs. (8) and (9)] can calculated from the horizontal wind components $u_{i j}$ and $v_{i j}$ as

$$
\begin{aligned}
\boldsymbol{v}_{e}^{i j}(k) & =a_{k} u_{i j}+b_{k} \boldsymbol{v}_{i j}, \\
& =\frac{a_{k} b_{j}-a_{j} b_{k}}{\Delta_{i j}} v_{a}(i)+\frac{a_{i} b_{k}-a_{k} b_{i}}{\Delta_{i j}} v_{a}(j), \\
& =\frac{\Delta_{k j}}{\Delta_{i j}} v_{a}(i)+\frac{\Delta_{i k}}{\Delta_{i j}} v_{a}(j) .
\end{aligned}
$$

Afterward, $\boldsymbol{v}_{e}^{i j}(k)$ is compared with the measured component of receiver $k$.

Assuming $\epsilon$ to be the measurement error of the Doppler velocity, which is equal for each receiver. $\{$ i.e., $\epsilon=$ $\left.\delta\left[v_{a}(i)\right]=\delta\left[v_{a}(j)\right]=\delta\left[v_{a}(k)\right]\right\}$, the error between $v_{a}^{i j}(k)$ and the measured component of receiver $k$ becomes

$$
\begin{aligned}
\delta & {\left[v_{e}(k)-v_{e}^{i j}(k)\right] } \\
& =\delta\left[v_{e}(k)-\frac{\Delta_{k j}}{\Delta_{i j}} v_{e}(i)-\frac{\Delta_{i k}}{\Delta_{i j}} v_{e}(j)\right] \\
& =\delta\left[v_{e}(k)\right]+\delta\left[\frac{\Delta_{k j}}{\Delta_{i j}} v_{e}(i)\right]+\delta\left[\frac{\Delta_{i k}}{\Delta_{i j}} v_{e}(j)\right] \\
& =\epsilon+\left|\frac{\Delta_{k j}}{\Delta_{i j}}\right| \epsilon+\left|\frac{\Delta_{i k}}{\Delta_{i j}}\right| \epsilon=\frac{\left|\Delta_{i j}\right|+\left|\Delta_{k j}\right|+\left|\Delta_{i k}\right|}{\left|\Delta_{i j}\right|} \epsilon .
\end{aligned}
$$

The difference between $v_{e}(k)$ and $v_{e}^{i j}(k)$ must be of the same order of magnitude as the measurement errors with

$$
\left|v_{e}(k)-v_{e}^{i j}(k)\right| \leq \frac{\left|\Delta_{i j}\right|+\left|\Delta_{k j}\right|+\left|\Delta_{i k}\right|}{\left|\Delta_{i j}\right|} \epsilon,
$$




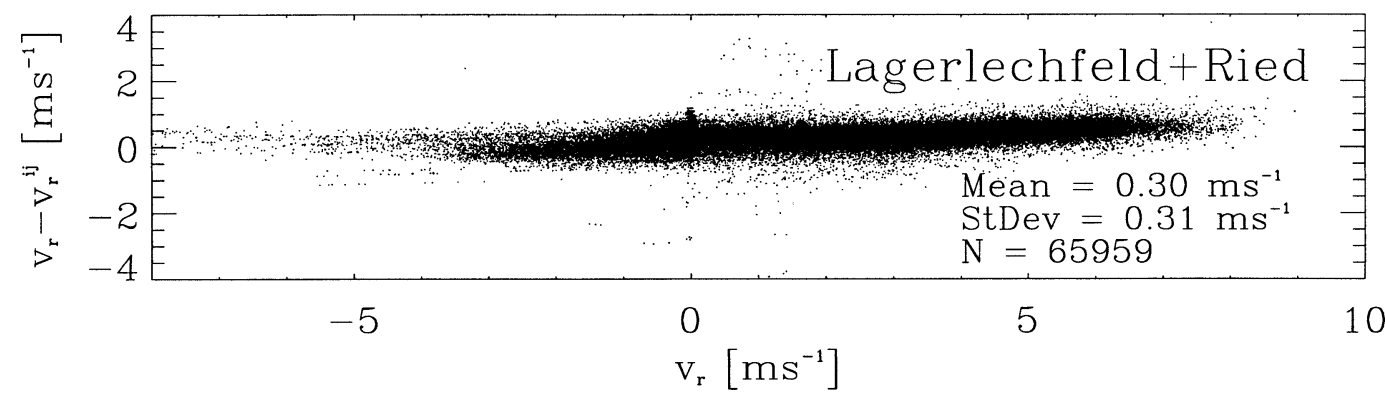

FIG. 3. Scatterplot illustrating the utilization of data consistency based on an internal check. The horizontal wind field $\left(u_{i j}, v_{i j}\right)$ was derived using Doppler information of the receivers at Lagerlechfeld $\left[v_{e}(i)\right]$ and Ried $\left[v_{e}(j)\right]$. From the horizontal wind field the radial velocity component of receiver OP, $v_{r}^{i j}$, is calculated and compared with the measured radial velocity, $v_{r}$. Mean value (Mean), std dev (StDev), and number of data points $(N)$ are labeled. The data were recorded during stratiform precipitation at 0730 UTC 19 Jun 2001.

otherwise the measured data are contaminated. An example of data consistency is given for the wind vector fields measured during a stratiform precipitation event in the morning hours of 19 June 2001 by a bistatic multiple-Doppler radar network. In that case all three receivers passed the internal check. The differences between the derived and measured radial velocity component of receiver OP $\left[v_{e}(k)=v_{r}\right]$, as illustrated in Fig. 3 , are mainly $\pm 2 \mathrm{~m} \mathrm{~s}^{-1}$ with a standard deviation of $0.31 \mathrm{~m} \mathrm{~s}^{-1}$ and a mean value of $0.30 \mathrm{~m} \mathrm{~s}^{-1}$. The horizontal wind components $\left(u_{i j}, v_{i j}\right)$ were derived from the components measured by the receivers at Lagerlechfeld and Ried. Figure 4 shows that 95\% (97\%) of the velocity differences are below $1 \mathrm{~m} \mathrm{~s}^{-1}$ for the receiver combination of Lichtenau-Ried (Lichtenau-Lagerlechfeld). Even $99 \%$ of the velocity differences are below $1 \mathrm{~m} \mathrm{~s}^{-1}$ for receiver combination of Lagerlechfeld-Ried. Figure 5 portraits the difference between the Doppler velocity measured by the Lichtenau receiver $\left[v_{e}(k)\right]$ and that reconstructed from the bistatic dual-

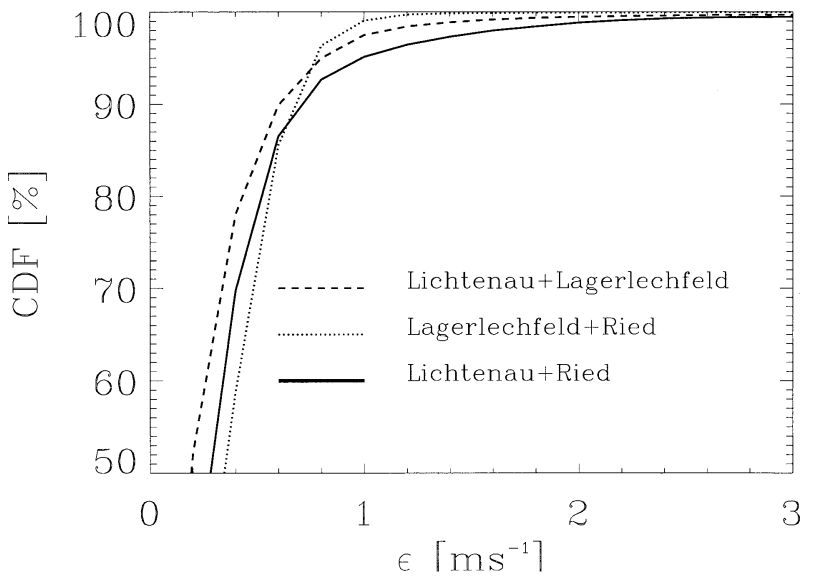

FIG. 4. Illustration of the empirical $\mathrm{CDF}(\%)$ and the measurement error, $\epsilon\left(\mathrm{m} \mathrm{s}^{-1}\right)$, for the Doppler velocities measured during stratiform precipitation at 0730 UTC 19 Jun 2001 . CDF is related to the velocity difference between the receiver OP and the combination of bistatic receivers (Lichtenau-Lagerlechfeld, Lagerlechfeld-Ried, LichtenauRied).
Doppler analysis using the receivers at OP $\left[v_{e}(i)\right]$ and Lagerlechfeld $\left[v_{e}(j)\right]$. The differences were on the order of $\pm 1 \mathrm{~m} \mathrm{~s}^{-1}$. Other combinations of received Doppler velocities gave similar differences for that stratiform precipitation event.

The consistency check succeeds when the differences for measurements within the multiple-Doppler area are within the measurement error of the Doppler velocity measurement. This means that the cumulative probability distribution function (CDF) should reach $80 \%$ at $1 \mathrm{~m} \mathrm{~s}^{-1}$. Although the sensitivity of bistatic receivers is less compared to monostatic radars, the quality of the measurement is the same when the signal-to-noise ratio is sufficient high. Major velocity differences occur, for example, when the bistatic receivers are not synchronized with the transmitting site. Sidelobe contamination

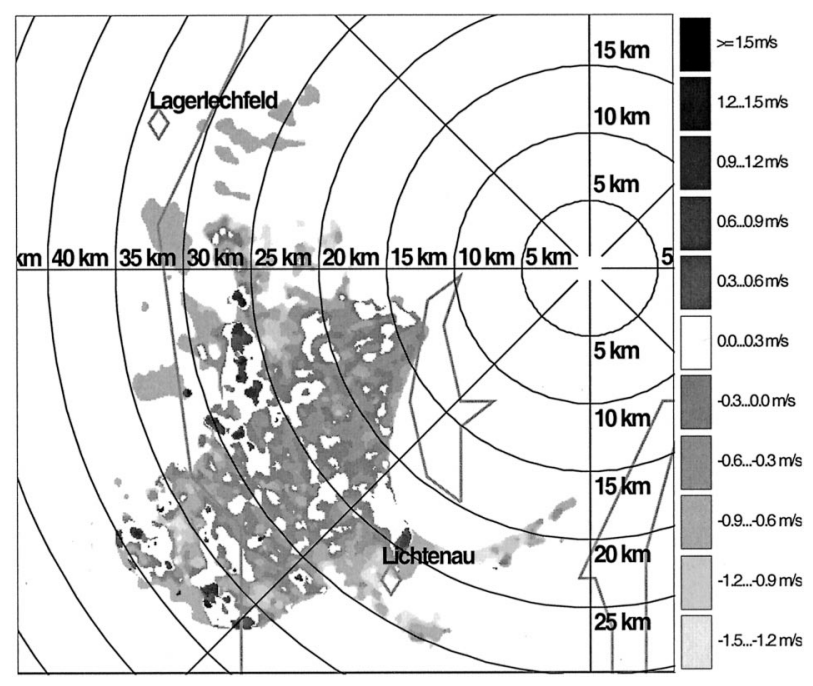

FIG. 5. PPI at $2^{\circ}$ elevation showing differences $\left(\mathrm{m} \mathrm{s}^{-1}\right)$ between the Doppler velocity measured by the receiver at Lichtenau and the reconstructed Doppler velocity for a stratiform precipitation event at 0730 UTC 19 Jun 2001. The reconstructed Doppler velocity was determined from the horizontal wind field (OP + Lagerlechfeld), which was then projected onto the direction measured by the receiver at Lichtenau. 


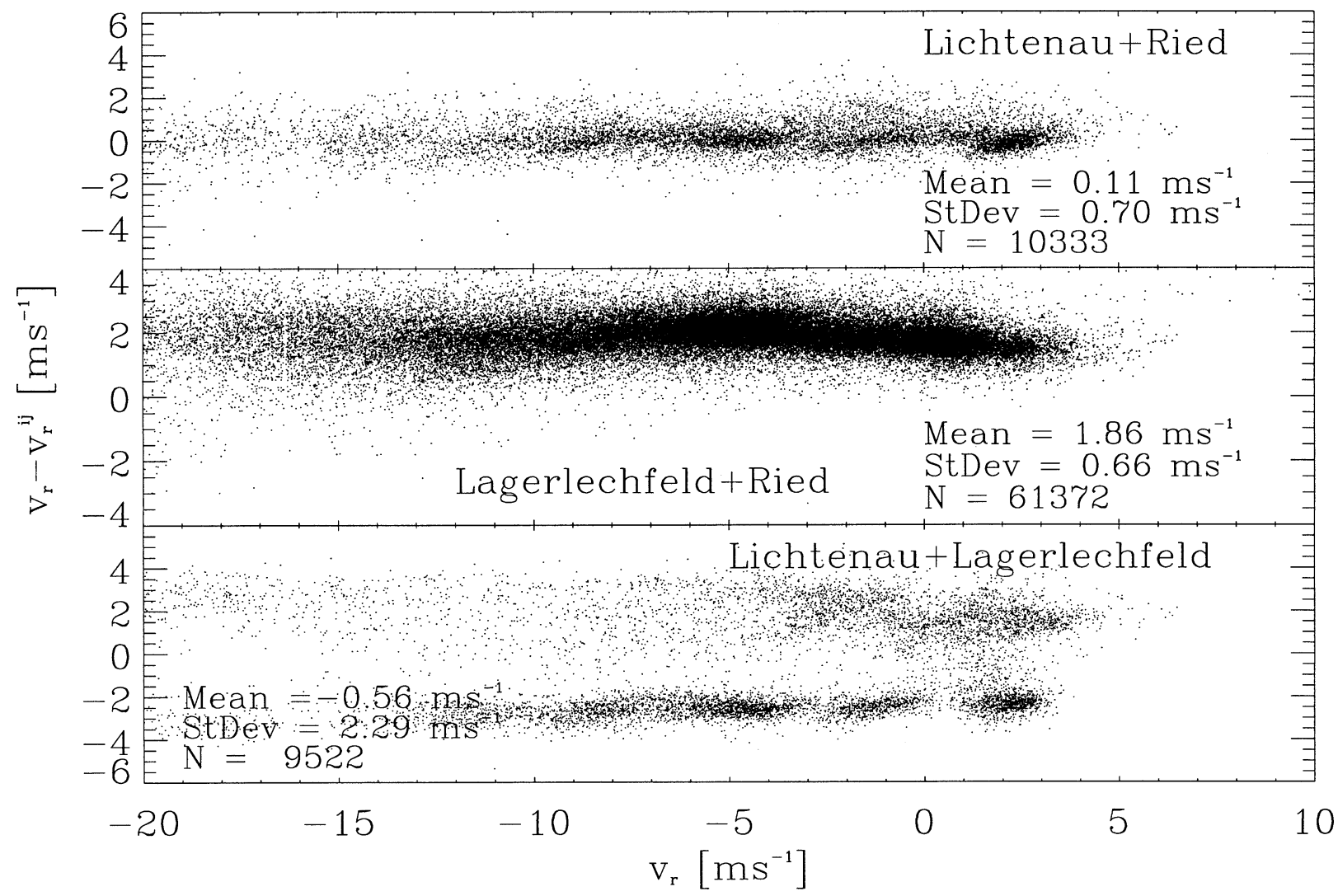

FIG. 6. Same as Fig. 3, but for the internal check that was applied to the data measured at 1556 UTC 9 Jul 2002. The horizontal wind field was derived using Doppler information of the receiver combinations $(i, j)$ Lichtenau-Ried, Lagerlechfeld-Ried, as well as LichtenauLagerlechfeld.

can also cause the internal check to fail. An example of data inconsistency is given for wind fields measured during a convective weather event at 1556 UTC 9 July 2002 (Figs. 6, 7, 8). While 99\% of the differences between the Lichtenau $\left[v_{e}(i)\right]$ and Ried $\left[v_{e}(j)\right]$ receiver measurements lie within the interval of $\pm 2 \mathrm{~m} \mathrm{~s}^{-1}$ when compared with the radial velocity, $v_{e}(k)=v_{r}$ (Fig. 6,

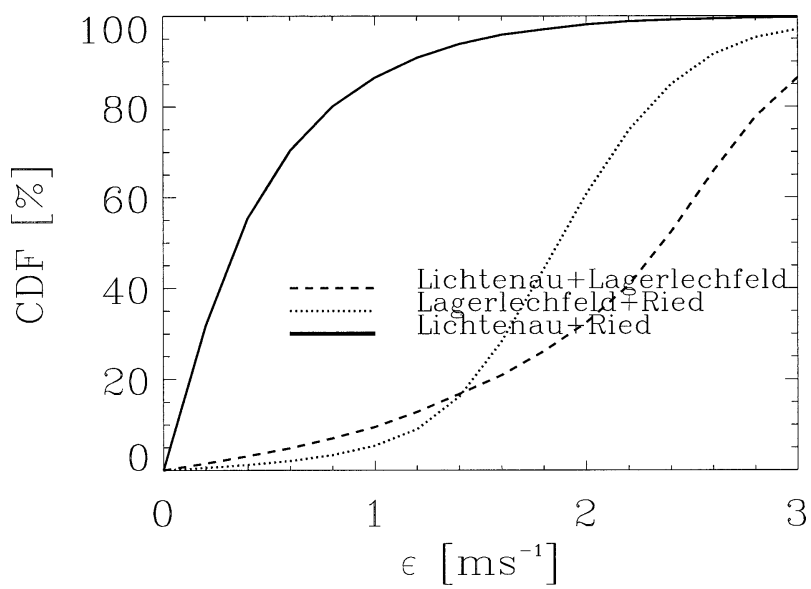

FIG. 7. Same as Fig. 4, but for the Doppler velocities measured at 1556 UTC 9 Jul 2002. upper part; Fig. 7, solid line), the combinations with the receiver at Lagerlechfeld fail (Fig. 6, middle and lower part; Fig. 7, dashed and dotted lines). Because sidelobe contamination was not observed, internal check failure can be attributed to asynchronous time and frequency measurements of the receiver at Lagerlechfeld compared to the transmitting radar at OP. Time and frequency measurements are synchronized to the monostatic radar at the Lichtenau and Ried receivers. A point-by-point comparison exhibited in Fig. 8, using the receiver at Ried $\left[v_{e}(k)\right]$ and the receiver combination at OP $\left[v_{e}(i)\right]$ and Lagerlechfeld $\left[v_{e}(j)\right]$, shows differences ranging from 4 to $10 \mathrm{~m} \mathrm{~s}^{-1}$. In this case, Doppler information from the receiver at Lagerlechfeld are not used for further data processing.

In order to identify $n$ incorrectly working receivers with this internal check, there has to be $n+3$ number of receivers covering the same area. Assuming only one receiver fails in a quadruple-Doppler network, the incorrectly operating receiver can be identified (cf. Fig. 6). Otherwise, if the number of receivers monitoring the same region is less than three, only inconsistency is detected but not the incorrectly working receiver. Because receiver synchronization and sidelobe contamination are the major error source from our experiences, 


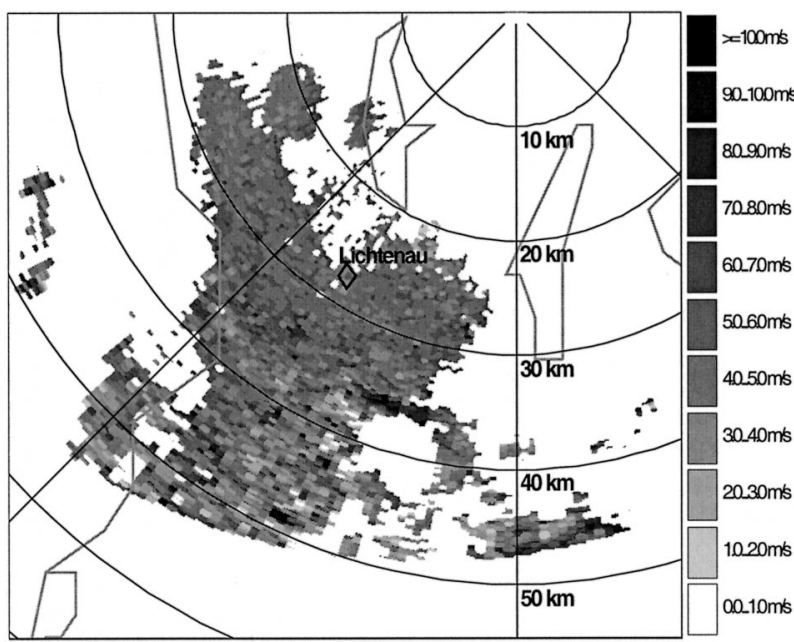

FIG. 8. PPI at $7^{\circ}$ elevation showing differences $\left(\mathrm{m} \mathrm{s}^{-1}\right)$ between the Doppler velocity measured by the receiver at Lagerlechfeld and the reconstructed Doppler velocity for a convective precipitation event at 1556 UTC 9 Jul 2002. The reconstructed Doppler velocity is determined from the horizontal wind field (OP + Ried), which is then projected onto the direction measured by the receiver at Lagerlechfeld.

the internal check is a fundamental algorithm within the automated evaluation tool. Data coming from incorrectly working receivers are excluded at that point in order to avoid misinterpretation within the further evaluation steps.

\section{c. The quality index fields}

The following algorithms weight the quality of each measurement with values ranging from 0 to 1 . A 1 corresponds to measurements with high quality, while lowquality measurements are flagged with 0 values. The results of each weighting algorithm can be merged to achieve an average quality-control field that reflects the confidence of each horizontal wind measurement.

In the following sections, three quality index fields are defined according to 1) the accuracy of the horizontal wind field, depending on the location of transmitter, target, and receiver, denoted as $F_{\text {stdev }} ; 2$ ) the amount of sidelobe contamination, denoted as $F_{\mathrm{VZ}}$; and 3 ) the fractional uncertainty of the wind field measurement, denoted as $F_{\text {frac }}$. Note that the standard deviation of the horizontal wind field, induced by the position of transmitter, target, and receivers, is fixed for a chosen experimental setup.

The influence of each quality index field on the average quality index field can be chosen according to the application of those quality-controlled wind vectors and the weather situation with the respective weights $W_{\text {stdev }}$, $W_{\nabla z}$, and $W_{\text {frac }}$. The quality fields can then be averaged to a single quality field using

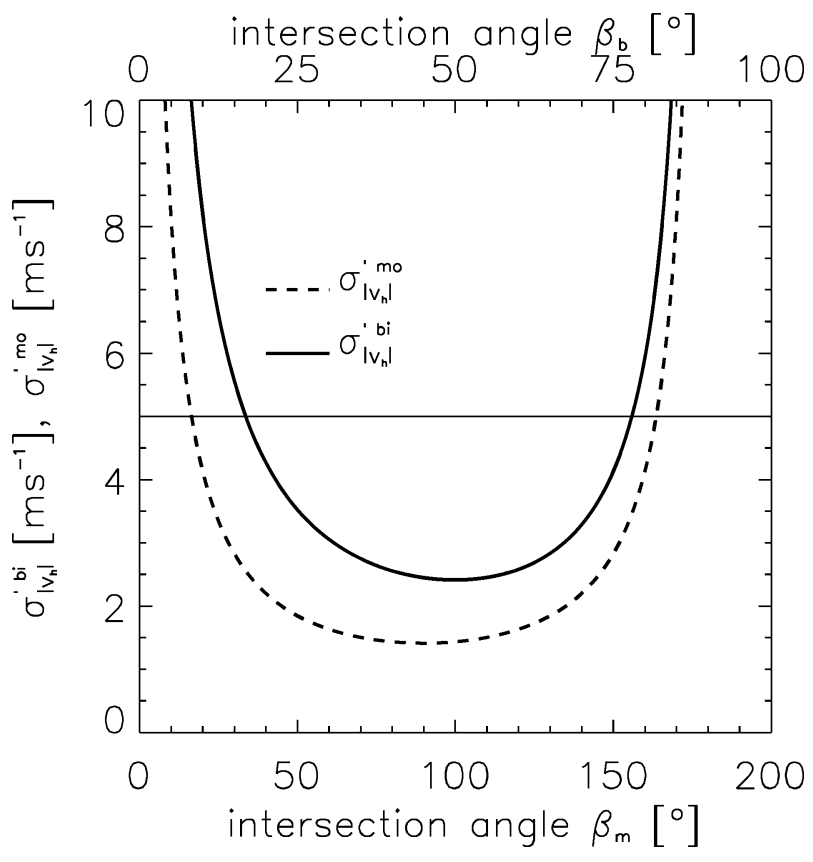

FIG. 9. Illustration of the dependency of the horizontal wind field's std dev measured by a monostatic dual-Doppler system, $\sigma_{\left|\mathbf{V}_{h}\right|}^{\prime}$, and a bistatic dual-Doppler system, $\sigma_{\left|\mathbf{V}_{h \mid}\right|}^{\prime \text { bi }}$, on the respective intersection angle, $\beta_{m}$ and $\beta_{b}$. The standard deviation of the dual-Doppler measurements is normalized by $1 \mathrm{~m} \mathrm{~s}^{-1}$, which can be assumed to be the instrumentation error of a receiver. Assuming an upper limit of the standard deviation to be $3 \mathrm{~m} \mathrm{~s}^{-1}$ (thin, solid horizontal line), the area is restricted by $25^{\circ} \leq \beta_{b} \leq 70^{\circ}$ within the bistatic dual-Doppler network, and by $20^{\circ} \leq \beta_{m} \leq 160^{\circ}$ for a monostatic dual-Doppler radar arrangement.

$$
\bar{F}=\frac{1}{C}\left(W_{\text {stdev }} F_{\text {stdev }}+W_{\mathrm{\nabla Z}} F_{\mathrm{\nabla} Z}+W_{\text {frac }} F_{\text {frac }}\right),
$$

where

$$
C=W_{\text {stdev }}+W_{\mathrm{vz}}+W_{\text {frac }} .
$$

\section{1) UTILIZING GEOMETRICAL ACCURACY}

The horizontal wind field is determined by at least two independent velocity components using a least squares estimation. The standard deviation of the horizontal wind field depends on the intersection angle between the two velocity components. Figure 9 illustrates the dependency of the standard deviation of the horizontal wind on the intersection angles, $\beta_{m}$ and $\beta_{b}$, for monostatic and bistatic radar networks, respectively. The highest accuracy is achieved at an intersection angle of about $50^{\circ}$ in the bistatic case (Takaya and Nakazato 2002) and of $90^{\circ}$ in the monostatic case. Horizontal wind fields cannot be determined at $\beta_{b}=0^{\circ}$ or $90^{\circ}$ or at $\beta_{m}$ $=180^{\circ}$, respectively. The standard deviation of the horizontal wind field, $\sigma_{\left|\mathbf{V}_{h}\right|}^{\prime}$, can be expressed by the quality index field $F_{\text {stdev }}$. Only the geometric location of the transmitter, receiver, and target is considered.

The highest accuracy in the horizontal wind field de- 


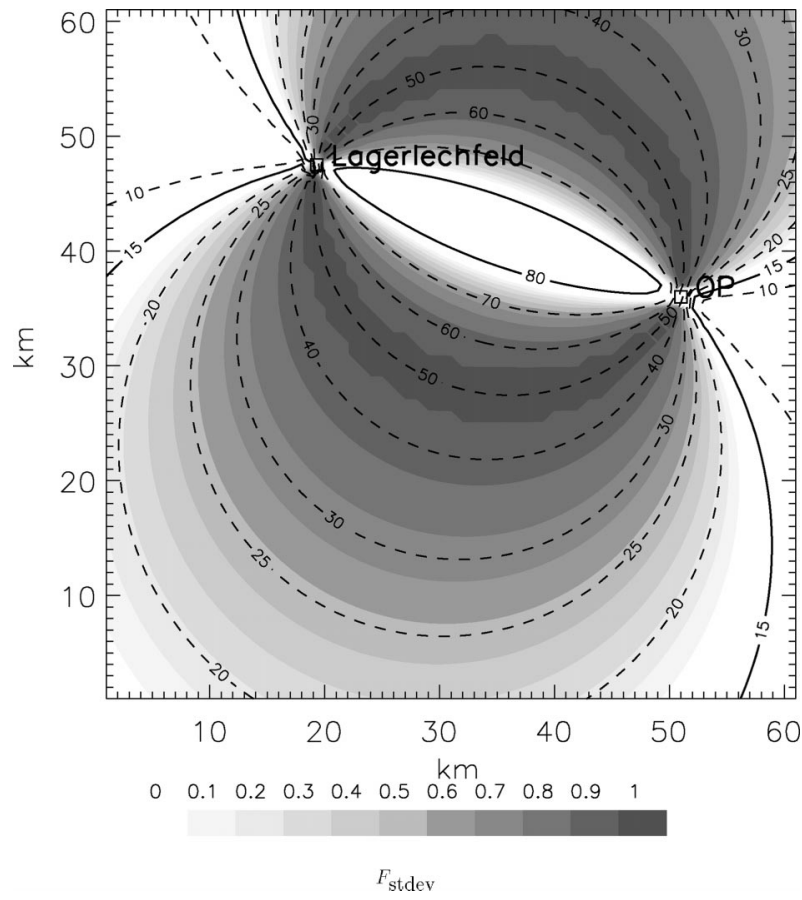

FIG. 10. Horizontal cross section at ground level of the quality index field $F_{\text {stdev }}$ superimposed on the intersection angle $\beta_{b}$ (dashed lines), for the bistatic dual-Doppler radar system consisting of OP and Lagerlechfeld; $F_{\text {stdev }}$ is only determined for an intersection angle ranging between $15^{\circ}$ and $80^{\circ}$ (thick, solid lines).

termination is expressed by $F_{\text {stdev }}=1$ at $\min \left(\sigma_{\left|\mathbf{v}_{h}\right|}^{\prime}\right)$, and the lowest accuracy by $F_{\text {stdev }}=0$. Because of the infinity of $\sigma_{\left|\mathbf{v}_{h}\right|}^{\prime}$ close to the baseline, the upper limit of $\sigma_{\left|\mathbf{V}_{h}\right|}^{\prime}\left[\max \left(\sigma_{\left|\mathbf{V}_{h}\right|}^{\prime}\right)\right]$ has to be chosen empirically in order to avoid $F_{\text {stdev }}$ from becoming infinitive. For a bistatic dual-Doppler radar system, the standard deviation reaches its minimum at a intersection angle of about $50^{\circ}$ with $\min \left(\sigma_{\left|\mathbf{V}_{h}\right|}^{\prime}\right)=2.42 \mathrm{~m} \mathrm{~s}^{-1}$, and $\max \left(\sigma_{\left|\mathbf{V}_{h}\right|}^{\prime}\right)$ is set empirically to $5.0 \mathrm{~m} \mathrm{~s}^{-1}$ (solid line in Fig. 9) ${ }^{2}$ at an intersection angle of $15^{\circ}$ or $80^{\circ}$. In the monostatic dual-Doppler case, the minimum reaches a value of $\min \left(\sigma_{\left|\mathbf{V}_{h}\right|}^{\prime}\right)=1.42 \mathrm{~m}$ $\mathrm{s}^{-1}$ at $\beta_{m}=90^{\circ}$, while $\max \left(\sigma_{\left|\mathbf{v}_{h}\right|}^{\prime}\right)$ is also set empirically to $5.0 \mathrm{~m} \mathrm{~s}^{-1}$. The definition of $F_{\text {stdev }}$ is given by

$$
F_{\text {stdev }}=\frac{\max \left(\sigma_{\left|\mathbf{v}_{h}\right|}^{\prime}\right)-\sigma_{\left|\mathbf{v}_{h}\right|}^{\prime}}{\max \left(\sigma_{\left|\mathbf{v}_{h}\right|}^{\prime}\right)-\min \left(\sigma_{\left|\mathbf{v}_{h}\right|}^{\prime}\right)} .
$$

A horizontal cross section of the distribution of the quality index field $F_{\text {stdev }}$ for the bistatic dual-Doppler radar system (POLDIRAD + Lagerlechfeld) superimposed on $\beta_{b}$ is shown in Fig. 10. Note that $F_{\text {stdev }}=0$ occurs in this example at $\beta_{b} \leq 15^{\circ}$ because the upperlimit standard deviation was set to $5 \mathrm{~m} \mathrm{~s}^{-1}$. For a different upper limit, $F_{\text {stdev }}$ changes respectively.

\footnotetext{
${ }^{2}$ The values are normalized by the standard deviation of Doppler velocity measurement achieved by a monostatic radar, which is assumed to be $1 \mathrm{~m} \mathrm{~s}^{-1}$
}

\section{2) UTILIZING SIGNAL QUALITY BASED ON THE REFLECTIVITY GRADIENT}

In this section, a simple approach is presented, focusing only on the amount of contamination on the bistatically measured data from the first sidelobe of the transmitting power pattern in relation to the weather situation. More details on this topic can be found in de Elia and Zawadzki (2000). The offset-fed paraboloid antenna installed at POLDIRAD has an antenna beam pattern as illustrated in Fig. 11. The main power is sent in the direction that the antenna is pointing. A secondary maximum of power appears at an angle of about $1.7^{\circ}$ in the azimuthal and about $4.5^{\circ}$ in the vertical direction from the first power maximum, with a reduction of about 32 and $37 \mathrm{~dB}$, respectively, compared to the main power. For a monostatic radar system, the scattered power is received with the same antenna power pattern, while in the bistatic case, the signal is received at a remote site by antennas with a wide angular aperture (i.e., horizontal aperture of about $60^{\circ}$, and a vertical aperture of either $8^{\circ}$ or $22^{\circ}$ ). The wide angular aperture is necessary to sample along the transmitted beam and, therewith, cover a large spatial area. Because the transmitted pulse travels with the speed of light, the bistatic antenna cannot be rotated along the propagation path. Low-gain antennas with wide angular apertures are more like to be affected by signal contamination, for example, multiple scattering, the first sidelobe, and integrated far-sidelobe coupling (Wurman et al. 1993; de Elia and Zawadzki 2000).

All signals arriving at the receiver at the same time are assigned to the area, where the main beam points, and to the corresponding sample time. Therefore, if an area is hit by a sidelobe of the transmitted power pattern, the receiver measures the power scattered by targets, which are illuminated by the main beam, plus the power scattered by targets, which are illuminated by the sidelobe, because both signals have the same propagation time. Depending on the transmitting antenna pattern the intensity reduction of the first sidelobe compared to the mainlobe can range between about 20 and $35 \mathrm{~dB}$ for typical weather radar systems. But also, the integrated energy emitted from the far sidelobes will be scattered toward the receiver. Typically the main lobe is about $50-70 \mathrm{~dB}$ above the far sidelobes, that is, radar data will be contaminated by that energy only if the reflectivity of the target exceeds about 50-70 dBZ (depending on the transmitting antenna pattern). This contamination becomes visible especially during convective weather events with isolated cells of strong reflectivity. If the far sidelobes hit the area of strong reflectivity the transmitted power is projected to the direction of the main beam, marking the ellipsoid of constant time delay with low reflectivity values. While the contamination caused by the first sidelobe can appear within all weather situations-within the bright band in stratiform precipitation, for instance- the integrated far sidelobe cou- 
(a)

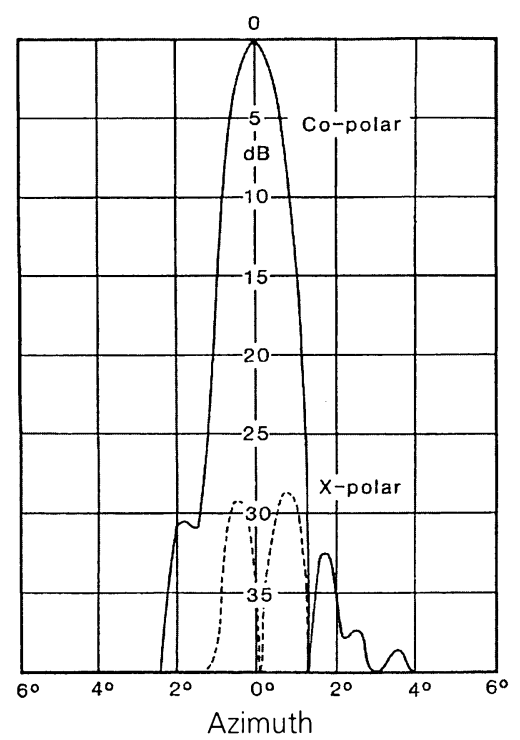

(b)

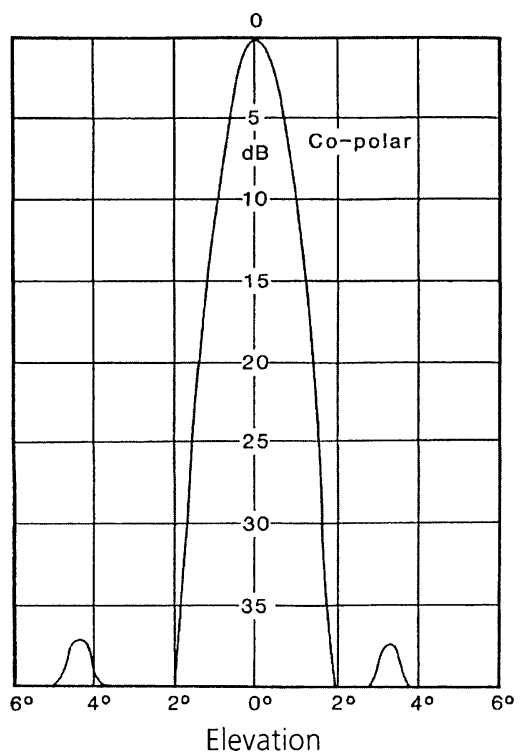

FIG. 11. One-way power pattern of the POLDIRAD antenna (dB) for transmitted vertical polarization. (a) Receiving power pattern sampled in an azimuthal direction with receiving vertical (Co-polar) and horizontal (X-polar) polarization. (b) Receiving power pattern sampled in the vertical direction with received vertical (Co-polar) polarization (Schroth et al. 1988).

pling appears mainly within convective situations where reflectivities of about 50-60 dBZ can be observed.

The quality control is limited to the first sidelobe coupling only because the amount on the far sidelobe is unknown for the POLDIRAD antenna and has, therefore, to be assumed. If the transmitting antenna pattern is completely known, a sidelobe contamination index as demonstrated by de Elia and Zawadzki (2000) can be used as the quality index field. de Elia and Zawadzki (2000) calculated the ratio between the power originating in the targets illuminated by the sidelobes and the total power consisting of the power coming from the target illuminated by the main lobe and the sidelobes.

This quality-control algorithm is only applied for data measured by a bistatic receiver, because those data are more likely to be contaminated by sidelobes of the transmitted radar due to the wide-beam receiving antennas. For narrow-beam antennas, sidelobe contamination can be normally ignored, because the signal transmitted through the weak sidelobe is also received by the weak sidelobe. Therefore, the reflectivity factor, $Z$, measured by the monostatic radar can be used as a reference to give information about the amount of sidelobe contamination. The probability that data measured by the bistatic receiver are contaminated by first sidelobes of the transmitted antenna pattern can be expressed by the gradient of the reflectivity factor, $\nabla Z=(\partial Z / \partial x, \partial Z / \partial y, \partial Z /$ $\partial z)$. The upper-limit value of the reflectivity factor gradient is derived from the beam pattern of the transmit- ting antenna at POLDIRAD, as illustrated in Fig. 11. Here, the first sidelobe of the transmitted beam pattern occurs at about $1.7^{\circ}$ away from the main beam in the azimuthal direction, with a reduction of about $32 \mathrm{~dB}$ when compared to the main lobe. In the vertical direction, the first sidelobe of the receiving power pattern occurs at $3.5^{\circ}$ and $4.5^{\circ}$ with a reduction of $37 \mathrm{~dB}$. Thus, assuming a uniform receiving beam pattern of the bistatic antenna, the gradient of the reflectivity factor has to be about $30 \mathrm{dBZ}$ per $1.7^{\circ}$ in order to measure the same signal intensity from the sidelobe as that obtained from the main lobe by the bistatic receiver. Because the horizontal wind together with the quality index field and the reflectivity factor field is located on a Cartesian grid, an analogous $30 \mathrm{~dB} \mathrm{~km}^{-1}$ is assumed $\left(1.7^{\circ}\right.$ at $30 \mathrm{~km}$ range correspond to $0.8 \mathrm{~km}$ ).

The absolute value of the reflectivity factor gradient can be expressed by the quality field $F_{\nabla Z}$, with

$$
F_{\nabla Z}=\frac{1-\left[\sqrt{\left(\frac{\Delta Z}{2 \Delta x}\right)^{2}+\left(\frac{\Delta Z}{2 \Delta y}\right)^{2}+\left(\frac{\Delta Z}{2 \Delta z}\right)^{2}}\right]}{\left(30 \mathrm{dBZ} \mathrm{km}^{-1}\right)} .
$$

At $F_{\mathrm{\nabla Z}}=0$, the amount of sidelobe contamination is very high, while at $F_{\nabla z}=1$, sidelobe contamination hardly occurs. As an example, the horizontal distribution of $F_{\nabla z}$ is analyzed in Fig. 12 for the reflectivity factor field of a simulated convective cell. The impact of $F_{\mathbf{v Z}}$ on the averaged quality index field is set by the weight- 


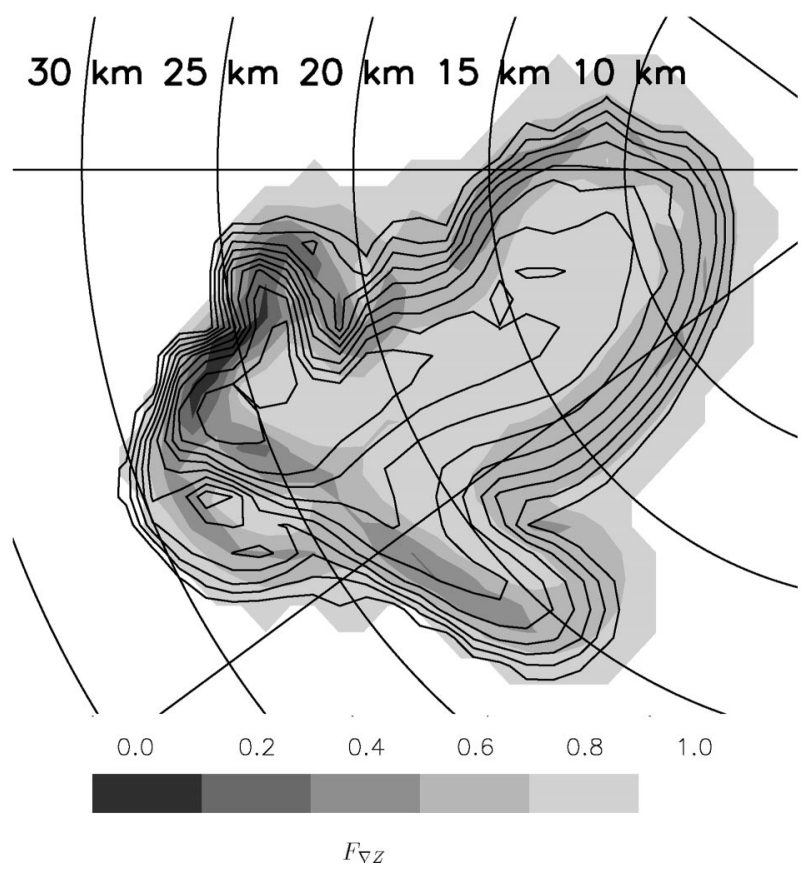

FIG. 12. Horizontal cross section of $F_{\mathrm{vz}}$ calculated for the simulated idealized convective cell. The isolines indicating the reflectivity factor are plotted every $5 \mathrm{dBZ}$, starting at the outside of the cell with the 5-dBZ isoline.

ing factor $W_{\nabla z}$. The weight $W_{\nabla z}$ should be set to 1 during all weather situations because of the probability of sidelobe contamination within the bright band of stratiform precipitation or transition zones between strong and weak reflectivity during convective situations.
3) UTILIZING SIGNAL QUALITY BASED ON FRACTIONAL UNCERTAINTY

Remote sensing with radar implies absolute measurements of the velocity component with constant error variances. Therefore, the fractional uncertainty field, $\sigma_{\left|\mathbf{v}_{h}\right|}^{\prime} /\left|\mathbf{V}_{h}\right|$, is used to give information about the percentage accuracy of the horizontal wind field measurement. The quality index field $F_{\text {frac }}$, related to the wind field variability, is derived linearly from $\sigma_{\left|\mathbf{v}_{h}\right|}^{\prime} /\left|\mathbf{V}_{h}\right|$ using

$$
F_{\text {frac }}= \begin{cases}1-\frac{\sigma_{\left|\mathbf{v}_{h}\right|}^{\prime}}{\left|\mathbf{V}_{h}\right|} & \text { for } \sigma_{\left|\mathbf{V}_{h}\right|}^{\prime}<\left|\mathbf{V}_{h}\right| \\ 0 & \text { for } \sigma_{\left|\mathbf{v}_{h}\right|}^{\prime} \geq\left|\mathbf{V}_{h}\right| .\end{cases}
$$

For $\sigma_{\left|\mathbf{V}_{h}\right|}^{\prime} \ll\left|\mathbf{V}_{h}\right|, F_{\text {frac }}$ approaches the value of one. Figure 13 shows the horizontal distribution of $F_{\text {frac }}$ for the simulated convective cell. The horizontal wind field of the simulated convective cell (Fig. 13a) is used to calculate $F_{\text {frac }}$ for the dual-Doppler configuration consisting of POLDIRAD and the receiver at Lagerlechfeld. Because the wind velocity measurement is an absolute measurement, low wind speeds create low values of $F_{\text {frac }}$. In addition, high standard deviations, for example, close to the baseline or in the quasi-monostatic area, cause low values of $F_{\text {frac }}$. The influence of $F_{\text {frac }}$ on the average quality-control field is controlled by the weight of $W_{\text {frac }}$.

The weight of this quality index field on the average quality index field should be set low within those weather conditions having high wind shear. For example, as illustrated in Fig. 13b at an azimuth angle of $200^{\circ} \leq$ (a)

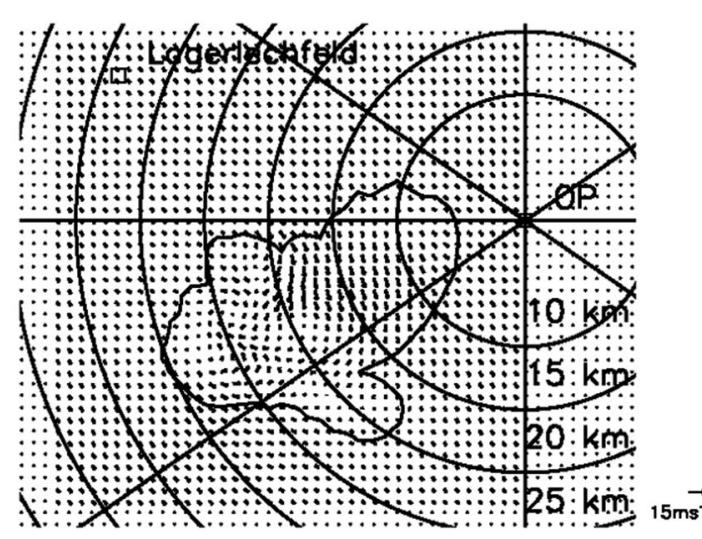

(b)

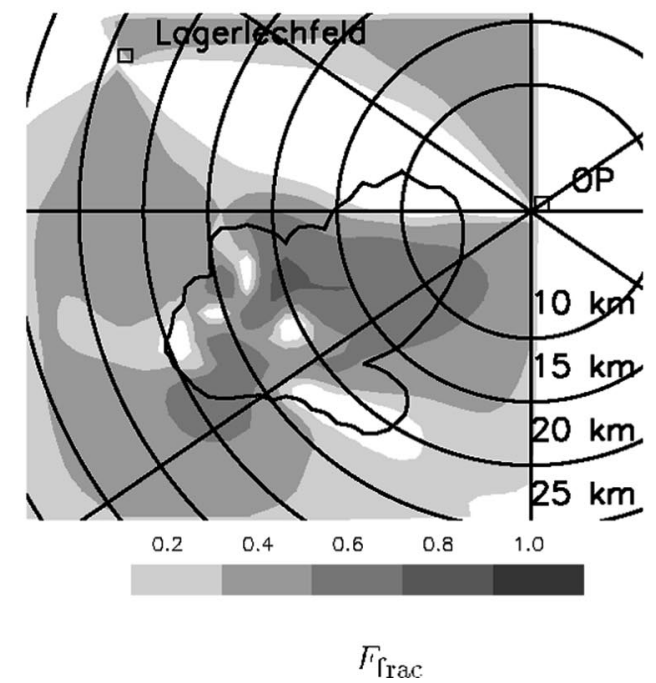

FIG. 13. Horizontal cross section of (a) the horizontal wind vector of a simulated convective cell and (b) the respective quality index field $F_{\text {frac }}$. The $5-\mathrm{dBZ}$ isoline indicating the shape of the convective storm is marked. 


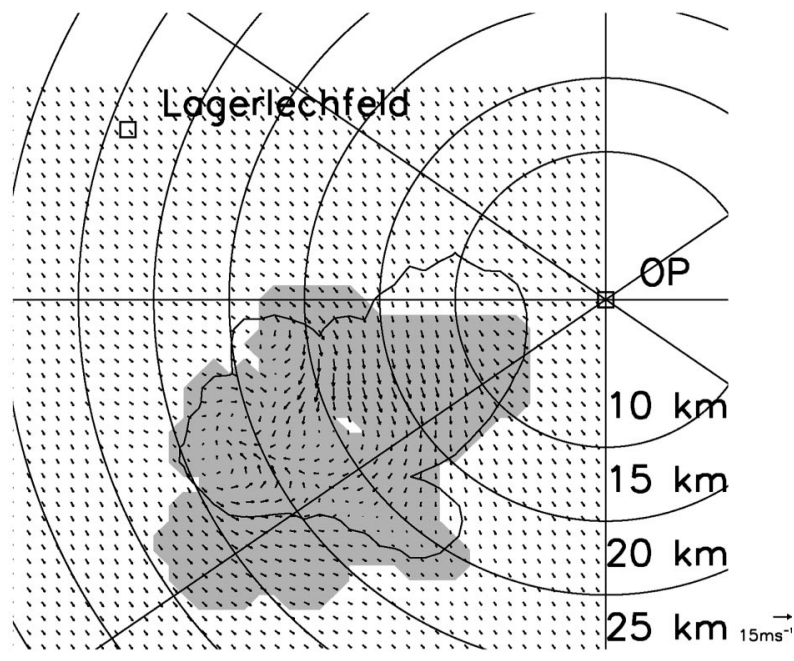

FIG. 14. Horizontal cross section of the horizontal wind vector (m $\mathrm{s}^{-1}$ ) within a convective storm. The area with wind shear (direction and absolute value) higher than mean flow is marked. The 5-dBZ isoline indicating the shape of the storm is marked.

$\phi_{t} \leq 225^{\circ}$ and at a range of $r_{t}^{\prime}=20-25 \mathrm{~km}$, the wind speed is very weak and causes $F_{\text {frac }}$ to be close to 0 , even though $\sigma_{\left|\mathbf{v}_{h}\right|}^{\prime}$ is low and the wind might represent realistic conditions within this area. To avoid a wrong interpretation of $F_{\text {frac }}, W_{\text {frac }}$ should be set high during stratiform precipitation with wind speed values larger than $5 \mathrm{~m} \mathrm{~s}^{-1}$ and low during situations with high wind shear, for example, convective situations.

\section{d. Utilizing data consistency based on a persistence check}

The data persistence check algorithm (referred to hereinafter as DCPC) should help to identify regions where wind shear is higher than the mean flow. The regions identified are then tracked in space by means of wind fields at different elevations and different time steps (e.g., successive volume scans). When high wind shear is identified at different levels and/or in the successive volume scans, the wind shear can be related to atmospherical processes. Otherwise, the perturbation originated either by meteorological, biological, or other targets is removed.

The persistence check is carried out as follows. The standard deviation in the horizontal wind velocity, $\sigma_{\left|\mathbf{v}_{h}\right|}$, and direction are calculated for each wind vector. The area is marked when the difference between the mean value of the height level, $\overline{\mathbf{V}_{h}}$, minus the $i$ th measured horizontal wind vector, $\mathbf{V}^{i}$, is larger than the vector standard deviation, $\sigma_{\mathbf{v}_{h}}$. For the horizontal wind velocity, this is given as

$$
\sigma_{\left|\mathbf{v}_{h}\right|}-\left|\overline{\mathbf{V}_{h}}-\mathbf{V}^{i}\right| \leq 0 .
$$

Figure 14 shows an example of how this algorithm is applied using the horizontal wind field of the simulated convective storm. The shape of the convective storm is indicated by the $5-\mathrm{dBZ}$ isoline. The wind shear within the storm is much higher than the surrounding airflow. Therefore, the area having high wind shear is marked. Note that if the area marked gray is present in the successive volume scans, as well as at different elevations, the marked area can then be identified as a signal that is related to meteorological processes. Otherwise, wind vectors within this area will be removed.

\section{Horizontal wind fields measured by bistatic Doppler radar network}

In this section results from the automated evaluation tool for bistatic wind field measurements are presented. First the NCP criterion (cf. section 4a) is applied to the raw Doppler velocity before the Doppler velocity data are internally checked (cf. section 4b). Velocities passing the internal check were used as input data for the constraining model (Protat and Zawadzki 1999) in order to retrieve the three components, $u, v$, and $w$, of the wind vector at the same time as the quality index field (as described in section 4c). At the end the data were utilized for consistency based on the persistence check (cf. section $4 d$ ).

\section{a. Stratiform precipitation in a frontal system (cases 1, 2)}

Two cold frontal systems with stratiform precipitation passed over southern Germany from the northwest on 2 February 2000 and 10 April 2001, respectively. On both days the prefrontal wind was mainly from southwesterly and westerly directions, ranging between 10 and $15 \mathrm{~m} \mathrm{~s}^{-1}$ and increasing and veering more to a westerly flow as the cold front approached. On 2 February 2000 data were collected by POLDIRAD and the receiver at Lagerlechfeld. On 10 April 2001, the bistatic Doppler radar network consisted of POLDIRAD and the receivers at Lagerlechfeld and Lichtenau. Both datasets were interpolated onto a Cartesian grid with a horizontal resolution of $500 \mathrm{~m}$ and a vertical resolution of $250 \mathrm{~m}$, starting at $600 \mathrm{~m} \mathrm{MSL}$, that is, at the height of POLDIRAD, up to a height of $2.85 \mathrm{~km} \mathrm{MSL}$.

Figure 15 shows the horizontal wind vector underlaid by the vertical velocity, the corresponding average quality index field, and the monostatic radar's reflectivity factor field measured during the frontal passage at 1708 UTC 2 February 2000, respectively. The frontal system was located southwest (SW) of OP (between $220^{\circ} \leq \phi_{t}$ $\leq 230^{\circ}$ ), heading southeast. In Fig. 15a, a perturbation can be detected SW of OP with a slight change in wind direction [from SW to west (W)], with prefrontal ascending and postfrontal descending air. Meteorological structures in the vertical velocity were visible within the observation area, and the overall structure of the vertical motion associated with a frontal passage can be verified with a light postfrontal updraft motion and a prefrontal downward motion. The averaged quality index field of 
(a)

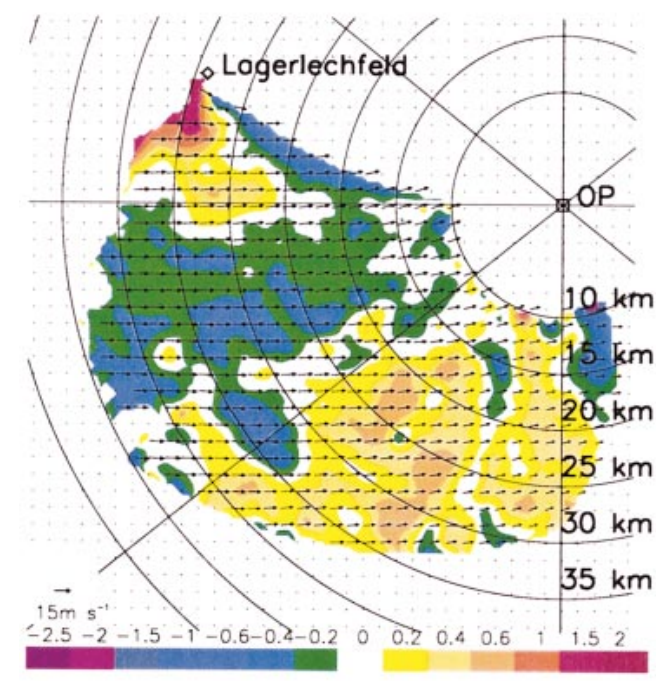

$\mathrm{w}\left[\mathrm{m} \mathrm{s}^{-1}\right]$ (b)

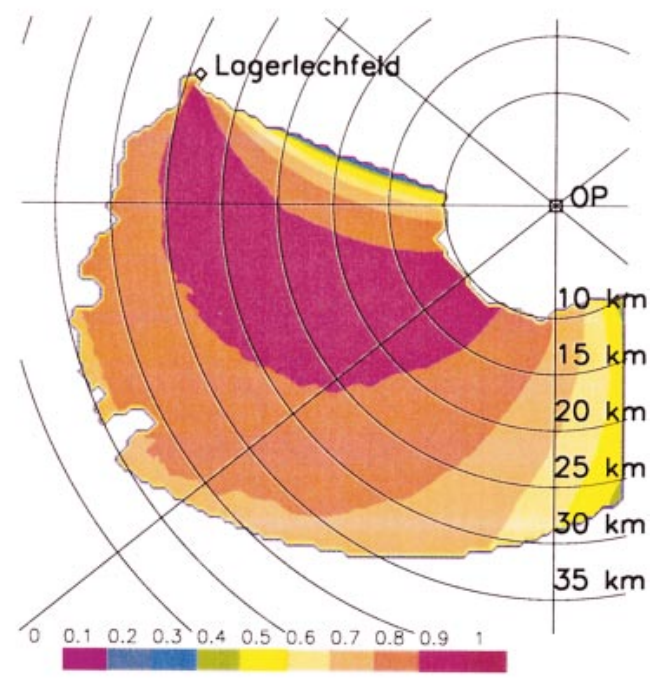

$\bar{F}$

(c)

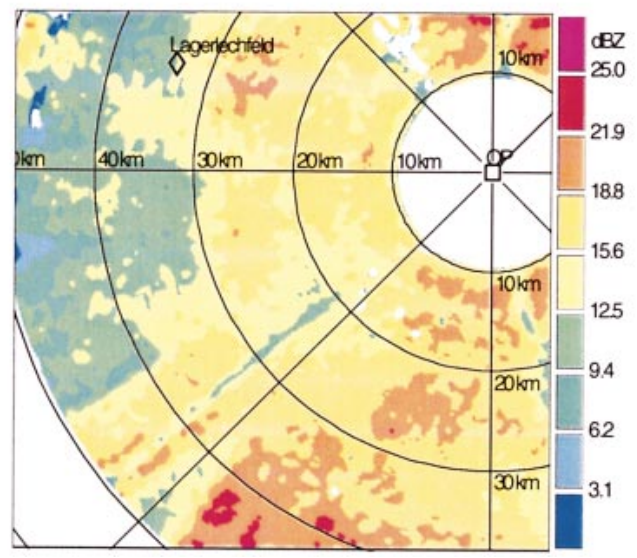

FIG. 15. Horizontal cross section at a height of 1.6 km MSL of the (a) retrieved horizontal wind vector underlaid by vertical velocity $\left(\mathrm{m} \mathrm{s}^{-1}\right.$ ), (b) the respective quality index field of the horizontal wind, and (c) the reflectivity factor field (dBZ) measured at 1708 UTC 2 Feb 2000 by the monostatic radar. Data were sampled by the receivers at $\mathrm{OP}$ and Lagerlechfeld. Upward motions are indicated by positive values, and downward motions are shown by negative values. For clarity of display, the wind vector are plotted with a horizontal resolution of $1.5 \mathrm{~km}$ (horizontal resolution of the wind retrieval is $500 \mathrm{~m}$ ).

the horizontal wind vector at $1708 \mathrm{UTC}$ is exhibited in Fig. 15b. The weights and algorithms are applied according to Table 1. In this case, which shows a weather situation with a relatively homogeneous flow, the quality index field is dominated by the accuracy of the horizontal wind field determination (cf. Fig. 10). All wind vectors with $\bar{F} \leq 0.5$ are used to determine the vertical velocity (cf. Figs. 15a and 15b). Vertical motion can also be verified by the reflectivity factor field indicating high reflectivities associated with upward motion and lower reflectivity values in areas with downward motion (cf. Fig. 15c). 
TABLE 1. An overview of the parameters set within the quality-control scheme that are applied for the respective instrumentation and weather situation.

\begin{tabular}{lcccc}
\hline \hline $\begin{array}{c}\text { Quality-control } \\
\text { scheme }\end{array}$ & $\begin{array}{c}\text { Stratiform precipitation: } \\
\text { case 1, two receivers } \\
\text { (bistatic Doppler radar) }\end{array}$ & $\begin{array}{c}\text { Stratiform precipitation: } \\
\text { case 2, three receivers } \\
\text { (bistatic Doppler radar) }\end{array}$ & $\begin{array}{c}\text { Convective precipitation: } \\
\text { case 3, two receivers } \\
\text { (bistatic Doppler radar) }\end{array}$ & $\begin{array}{c}\text { Stratiform precipitation: } \\
\text { case 4, two transceivers } \\
\text { (monostatic Doppler radar) }\end{array}$ \\
\hline SQI & Yes & Yes & Yes & Applied in signal processor \\
DCIC & No & Yes & 1 & 1 \\
$W_{\text {stdev }}$ & 1 & 1 & 1 & 0 \\
$W_{\text {vZ }}$ & 1 & 1 & 0 & 1 \\
$W_{\text {frac }}$ & 1 & 1 & Yes & Yes \\
DCPC & Yes & Yes & \\
\hline
\end{tabular}

Figure 16 illustrates the vertical wind field superimposed on the horizontal wind vector field, the corresponding average quality index field of the horizontal wind, and monostatic radar's reflectivity factor field at 1327 UTC 10 April 2001, respectively. The 3D wind field represents the time before the frontal passage, which is characterized by southwesterly flow and upward motion ranging between $\pm 0.4 \mathrm{~m} \mathrm{~s}^{-1}$. The influence of an overdetermined equation system for the horizontal wind field calculation on the averaged quality index field can be seen clearly when comparing Figs. $15 \mathrm{~b}$ and $16 \mathrm{~b}$. According to the quality index field (Fig. $16 \mathrm{~b})$, the confidence in the horizontal wind field is high within the whole observation area (triple Doppler area), with values from 0.6 on. The retrieved vertical velocity was characterized by relatively small up- and downward motions because of the low values of horizontal convergence. A horizontal convergence in the wind field was observed in the northern and southern parts, which could be linked to upward motion with values of $w$ up to $1 \mathrm{~m} \mathrm{~s}^{-1}$. As during the stratiform precipitation event on 2 February 2000 (cf. Fig. 15), the upward motion can be verified by high reflectivity values ranging between 20 and $40 \mathrm{dBZ}$ as illustrated in Fig. 16c. Downward motion appears in areas with low reflectivity values (cf. Figs. 16a and 16c).

\section{b. Convective weather situation (case 3)}

On the afternoon of the 3 May 2000, a convective system developed southwest of OP around 1400 UTC and stayed within the observation area for $1 \mathrm{~h}$.

The most active part of the system located southwest of OP (i.e., maximum reflectivity factor) consisted of a stationary cell located between $225^{\circ} \leq \phi_{t} \leq 280^{\circ}$ at a range of $15 \mathrm{~km} \leq r_{t}^{\prime} \leq 30 \mathrm{~km}$ as indicated by high values in the monostatic radar's reflectivity factor field, illustrated in Fig. 17. Volume scans were performed by POLDIRAD and the receiver at Lagerlechfeld. The wind field retrieval was performed in a $40 \mathrm{~km} \times 40 \mathrm{~km} \times$ $10 \mathrm{~km}$ domain and interpolated onto a Cartesian grid with a horizontal and vertical resolution of $500 \mathrm{~m}$. The retrieval domain consisted of 20 height levels, beginning at $600 \mathrm{~m}$ and reaching up to a height of $10.6 \mathrm{~km}$ MSL. The weights and algorithms are applied according to Table 1. Unfortunately, the DCIC algorithm to detect receiver synchronization problems and sidelobe contamination could not be applied because of the dual-Doppler estimation.

Figure 18 shows the retrieved horizontal wind vector underlaid by the vertical velocity and the corresponding quality index for the horizontal wind field at 1455 UTC at a height of 1.35 and $5.35 \mathrm{~km}$ MSL. The horizontal wind vector at the lower troposphere showed strong divergences and convergences in speed and direction with an easterly and westerly flow at $1.35 \mathrm{~km} \mathrm{MSL} \mathrm{(Fig.}$ 18a). Aloft, northwesterly to westerly winds dominated (Fig. 18c), with a noticeable confluence of the outflow on the southern part of the most active part of the system. The vertical velocity fields were characterized by a well-defined updraft located at $230^{\circ} \leq \phi_{t} \leq 270^{\circ}$ at a range of $15 \mathrm{~km} \leq r_{t} \leq 25 \mathrm{~km}$ from OP and a downdraft area south of it. The magnitude of the up- and downdraft was continuously increasing from ground level up to the top. Maximum values of $6 \mathrm{~m} \mathrm{~s}^{-1}$ at a height of 5.35 $\mathrm{km}$ MSL were reached in the updraft region and values of $-5 \mathrm{~m} \mathrm{~s}^{-1}$ at $5.35 \mathrm{~km} \mathrm{MSL}$ were found in the southern downdraft region (Fig. 18c). Both positive and negative divergences of the horizontal wind field were observed in the main updraft area.

The confidence in the horizontal wind field, $\bar{F}$, ranged between 0.5 and 0.9 . The vertical velocity is only calculated within an area with $\bar{F} \geq 0.8$ (cf. Figs. $18 \mathrm{~b}$ and $18 \mathrm{~d})$. Owing to the high variability in wind direction and speed, $W_{\text {frac }}$ is set to 0 . Furthermore, at lower elevations, high probability of sidelobe contamination was assumed because of the high gradient in the reflectivity factor and was, therefore, investigated separately. In Fig. 19 the bistatic reflectivity factor field is illustrated. If the data measured by the bistatic receiver were found to be contaminated by the transmitted secondary lobe, the bistatic reflectivity factor would be extended in the direction of the ellipsoid of the respective range gate (de Elia and Zawadzki 2000). As illustrated in Fig. 19, the maximum reflectivity factor expands in the radial direction from the bistatic receiver according to the received power pattern, not along the ellipsoid. The structures of bistatic reflectivity factor field are similar to the monostatic reflectivity field (cf. Figs. 17a and 19). The results showed low amounts of sidelobe contamination measured by the bistatic receiver during this convective situation. 
(a)

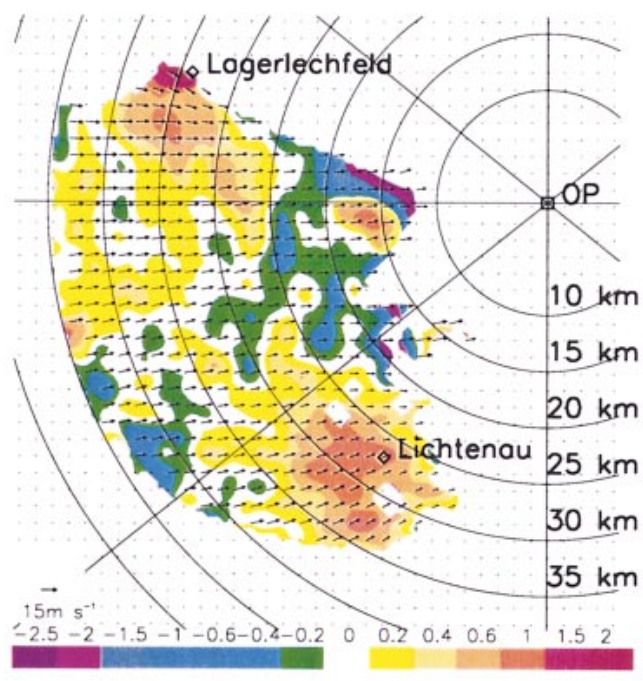

$\mathrm{w}\left[\mathrm{m} \mathrm{s}^{-1}\right]$
$\bar{F}$

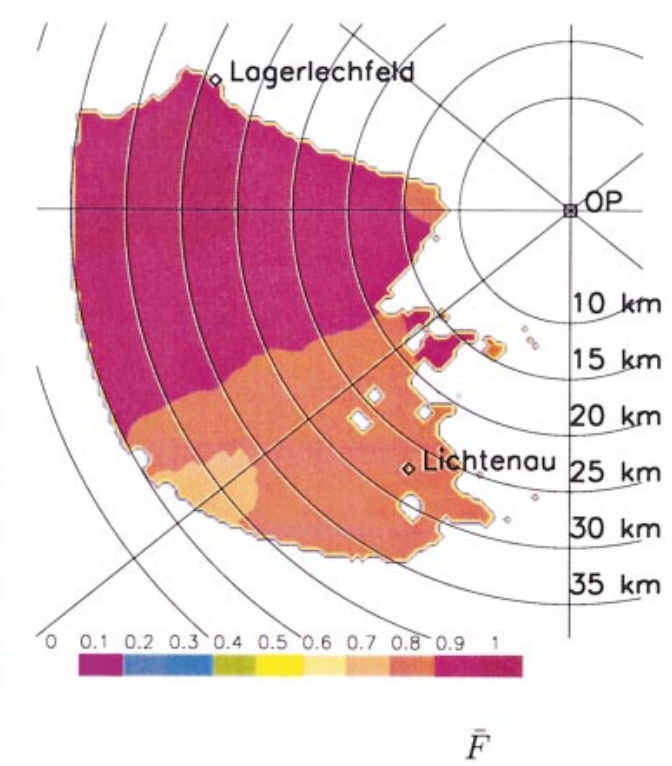

(b)

(c)

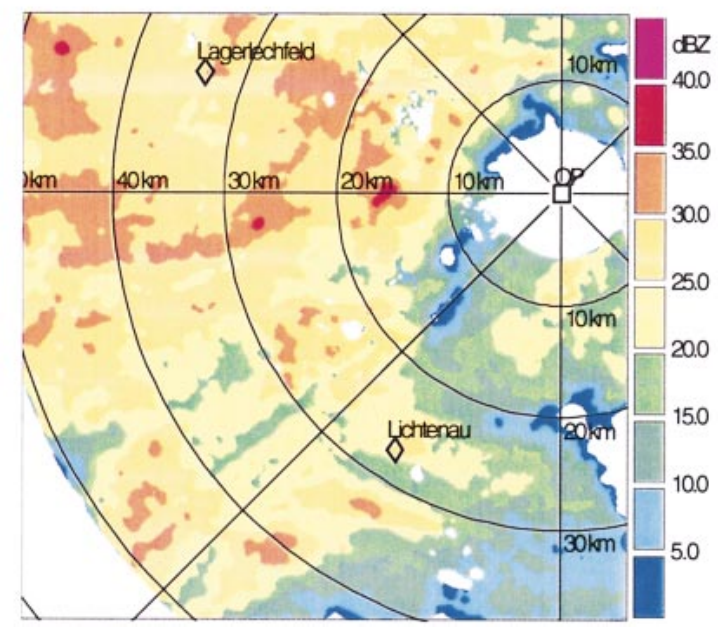

FIG. 16. Same as Fig. 15, but at 1327 UTC 10 Apr 2001. Data were sampled by the receivers at OP, Lichtenau, and Lagerlechfeld.

\section{Horizontal wind fields measured by monostatic dual-Doppler radar system}

Stratiform precipitation in a frontal system (case 4)

Between 1200 and 1530 UTC on 10 April 2001, stratiform precipitation was present within the observation area. During that time measurements by an independent monostatic Doppler radar at HP were achieved. This case was discussed for the bistatic Doppler radar system in detail in section 5a. The wind came mainly from the southwest, with velocities ranging between 10 and 14 $\mathrm{m} \mathrm{s}^{-1}$. A wind velocity gradient in an east-west direction over a length of $30 \mathrm{~km}$ occurred, having values of $14 \mathrm{~m} \mathrm{~s}^{-1}$ to the west and $6 \mathrm{~m} \mathrm{~s}^{-1}$ east of the observation area. The mean wind direction was about $260^{\circ}$, varying from $240^{\circ}$ to $265^{\circ}$. The horizontal wind vectors at 1347 
(a)

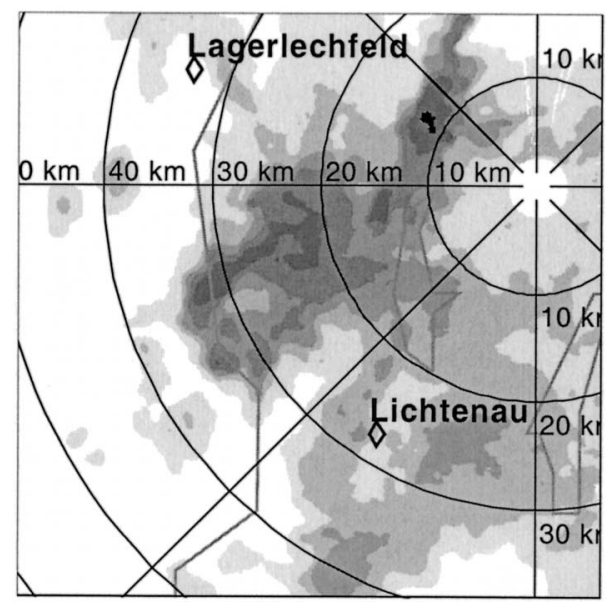

(b)

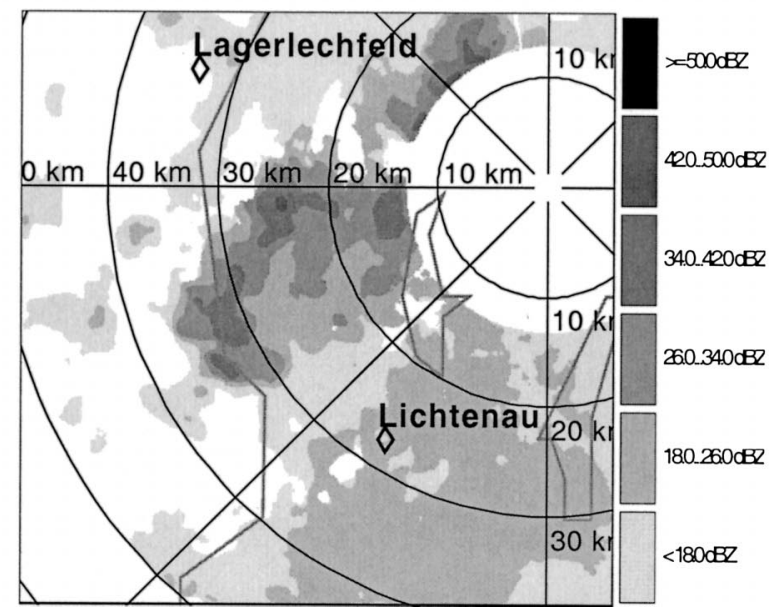

FIG. 17. Horizontal cross section of the reflectivity factor field (dBZ) measured by the monostatic Doppler radar at 1455 UTC 3 May 2000 at a height of (a) 1.6 and (b) $5.35 \mathrm{~km} \mathrm{MSL.}$

UTC are pictured in Fig. 20 (cf. wind field measured by the bistatic network at 1327 UTC is shown in Fig. 16a). Structures like, for instance, the area with winds coming from the SW at range of 35-60 km west of OP are observed by both the monostatic and bistatic Doppler radar network (cf. Figs. 16a and 20a). Close to the ground the wind direction varies between the westerly and southwesterly direction because of boundary effects. Within the turbulent area west of OP the reflectivity values increases up to $30-35 \mathrm{dBZ}$ in comparison with the surrounding area at 1327 UTC at a height of $1.6 \mathrm{~km}$ (Fig.16a). The turbulence wind structure at a $3-$ $\mathrm{km}$ height (Fig. 20a) can be related to turbulence at the frontal line. At an elevation of $6 \mathrm{~km}$ a uniform wind, as expected after a frontal passage coming from the southwest, was observed.

The horizontal wind field was determined using the radial velocities measured by the monostatic $\mathrm{C}$-band Doppler radars POLDIRAD and HP. The interpolation and wind synthesis was performed with the "SPRINT" and CEDRIC packages (Mohr et al. 1986).

The weights and algorithms are applied according to Table 1. Figure 20 illustrates the horizontal wind field underlaid by the averaged quality index field at 3 and $6 \mathrm{~km}$ MSL. The average quality index field $\bar{F}$ is dominated by $F_{\text {stdev }}$ and $F_{\text {frac }}$. The highest confidence of the measurement occurs in the areas with an intersection angle ranging between $80^{\circ}$ and $100^{\circ}$. The wind shear is higher at lower elevations, which causes the quality index field to be lower (cf. Figs. 20a and 20b). The amount of sidelobe contamination can be assumed to be 0 because both antenna patterns have a reduction of the first secondary sidelobe of $30 \mathrm{~dB}$. Therefore, the reflectivity gradient has to exceed a value of about $60 \mathrm{~dB} \mathrm{~km}^{-1}$ because the signal transmitted through the weak sidelobe is also received by the weak sidelobe.

\section{Summary and conclusions}

The horizontal wind field and signatures of the wind field (e.g., divergences, rotations, wind shear) can be detected with a bistatic Doppler radar as presented here for two stratiform and one convective precipitation event. While the horizontal wind is measured in real time, the quality control and the vertical wind retrieval are applied afterward. The three components of the wind vectors, $u, v$, and $w$, together with a confidence level for each horizontal wind field, is a great benefit for operational meteorology, for example, weather surveillance, hazard warnings, and nowcasting of weather phenomena.

For an operational application of the quality control, algorithms have to be applied differently and weights have to be set to be flexible, according to the weather situation and requested number of data points. Table 1 illustrates how the different quality index algorithms are applied according to the weather situation and the instrumentation. The decision criterion (denoted as SQI algorithm in Table 1) is applied to all bistatically measured data to remove noisy, spurious data in order to improve the efficiency of the succeeding algorithms and to remove artificial gradients that can be misinterpreted. Receiver synchronization problems and sidelobe contamination are the major error sources for bistatic measurements. Bistatic receivers that are not synchronized with the transmitting radar or data contaminated by the transmitting sidelobes can be detected and excluded from further processing using the data consistency in- 
(a)

\section{$\diamond$ Lagerlechfeld}

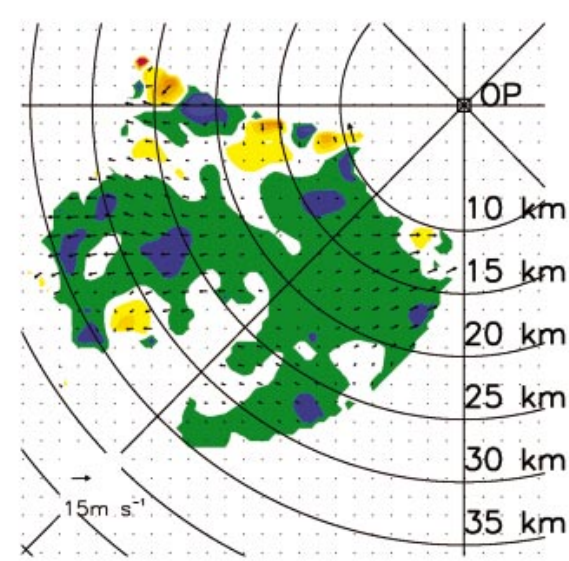

(c)

$\diamond$ Lagerlechfeld

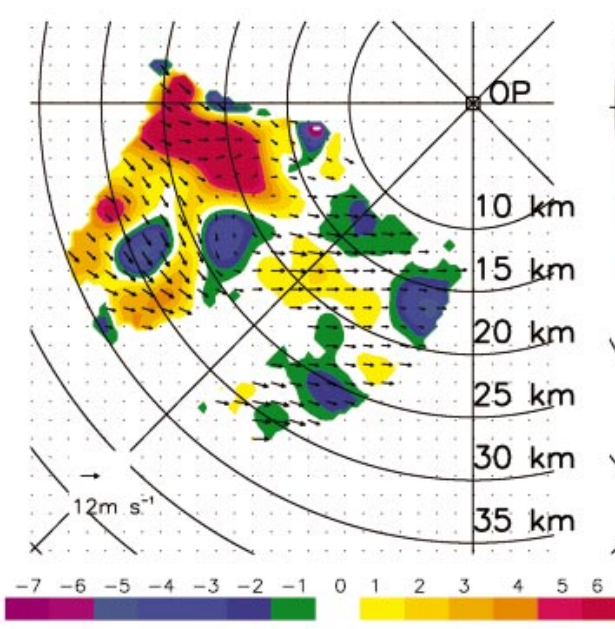

$$
\mathrm{w}\left[\mathrm{ms}^{-1}\right]
$$

(b)

$$
\text { - Lagerlechfeld }
$$

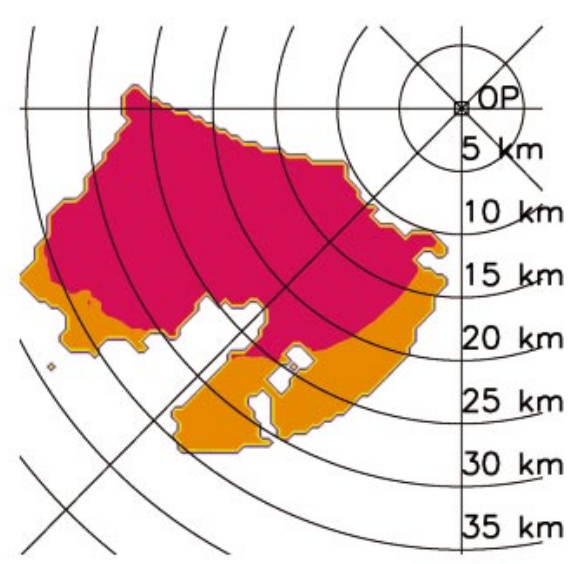

(d)

FIG. 18. Horizontal cross section of the horizontal wind vector $\left(\mathrm{m} \mathrm{s}^{-1}\right)$ at a height of (a) 1.35 and (c) $5.35 \mathrm{~km}$ MSL underlaid by the vertical velocity $\left(\mathrm{m} \mathrm{s}^{-1}\right)$ at 1455 UTC 3 May 2000. The respective quality index fields of the horizontal wind field are illustrated (b) for a height of 1.35 and (d) $5.35 \mathrm{~km}$ MSL. The horizontal wind was determined by the Doppler measurements of the receivers at OP and Lagerlechfeld. Upwinds are indicated by positive values; negative values are downwinds. For clarity of display, the wind vectors are plotted with a horizontal resolution of $1.5 \mathrm{~km}$ (horizontal resolution of the wind retrieval is $500 \mathrm{~m}$ ).

ternal check (DCIC). This internal consistency check can be applied also when at least two receiving antennas cover the same area. Because bistatic receivers are usually installed for a dual-Doppler application, it should be considered to install additional receiver. Alternatively, if several adjoint dual-Doppler systems are installed, rotating antennas can be used to temporally monitor the areas under investigation with three receiver systems. The antennas could be equipped with a motor, which enables them to be rotated once or twice per day to an area where at least three components of the wind field can be measured. During that time, it is possible to check for receiver synchronization and contamination from the transmitting sidelobes. Note that while using three receivers a failure can be detected, but the corresponding receiver cannot be identified.

The weight $W_{\text {stdev }}$ should be set high for multipleDoppler measurements within all weather situations be- 


\section{$\diamond$ Lagerlechfeld}

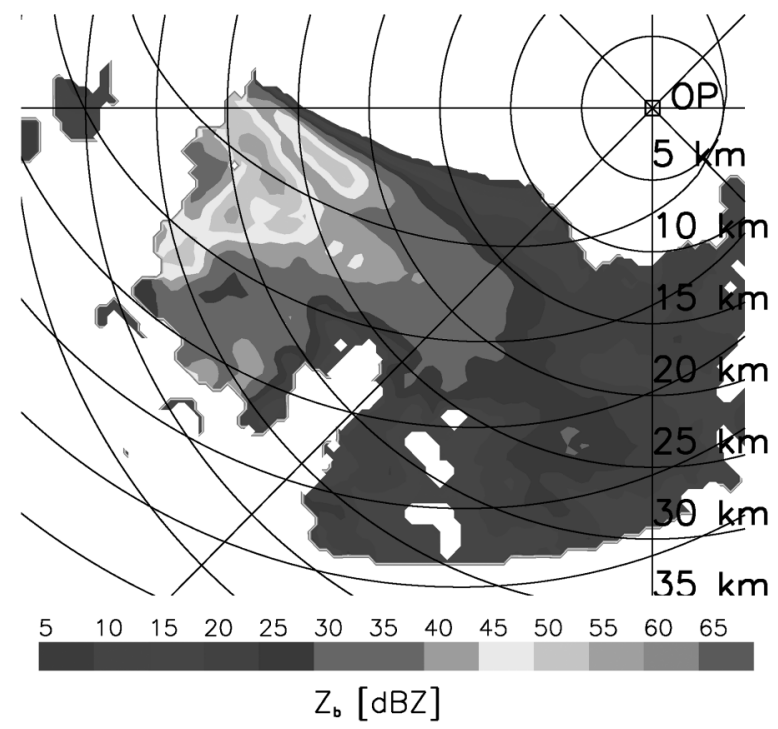

FIG. 19. Horizontal cross section of the bistatic reflectivity factor field normalized to the bistatic antenna pattern (dBZ) measured by the bistatic receiver at Lagerlechfeld at 1455 UTC 3 May 2000 at a height of $1.8 \mathrm{~km}$ MSL. Spheres and ellipsoids of constant delay are indicated. cause the geometrically induced standard deviation is independent from the weather situation but depends very much on the number of independent measurements. Those measurements can be achieved either by monostatic or bistatic Doppler radar networks or additional measurements from different instruments, for example, a wind profiler and lidar can be combined. The error variance depends always on the intersection angle between the independent measurement. Because sidelobe contamination can become a severe problem for bistatic measurements, the amount of contamination should be additionally quantified on behalf of the reflectivity fields within the quality index field. In this paper a computationally fast approach to estimate the amount of contamination that resulted from first sidelobe of the transmitted power is developed. An alternative to that approach, the sidelobe contamination index developed by de Elia and Zawadzki (2000), can be applied to $F_{\mathbf{v z}}$. Only for bistatic Doppler radar configuration (due to the wide aperture of the receiving antenna) should the weight $W_{\nabla z}$ be set to one during all-weather situations due to a possibility of sidelobe contamination within the bright band of stratiform precipitation or transition zones between strong and weak reflectivity during convective situations. To avoid a wrong interpretation of $F_{\text {frac }}, W_{\text {frac }}$ should be set high during stratiform precipitation with wind speed values larger than $5 \mathrm{~m} \mathrm{~s}^{-1}$ and set lower during situations with high wind shear, for example, convective situations independent from the instrumentation configuration. During high-wind-shear (a)

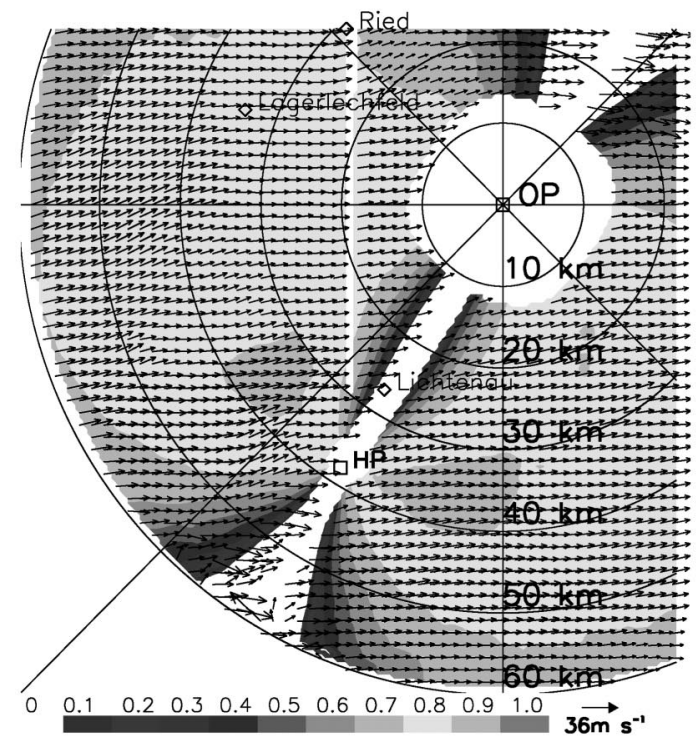

(b)

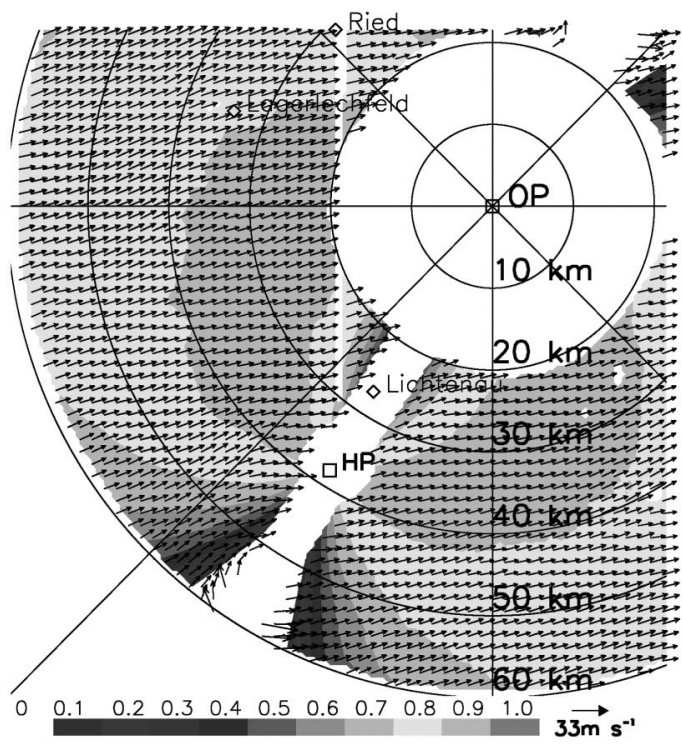

FIG. 20. Horizontal cross section of the horizontal wind vector $\left(\mathrm{m} \mathrm{s}^{-1}\right)$ at a height of (a) 3 and (b) $6 \mathrm{~km} \mathrm{MSL}$ underlaid by the averaged quality index field at 1347 UTC 10 Apr 2001. The horizontal wind was determined by the Doppler measurements of POLDIRAD and the monostatic Doppler radar at HP. For clarity of display, the wind vectors are plotted with a horizontal resolution of $1.5 \mathrm{~km}$. 
events erroneous wind vectors can be detected and removed using the DCPC algorithm. If DCPC detects areas with high wind shear, $W_{\text {frac }}$ is set to 0, owing to high wind shear. Again, wind measurements achieved by other remote sensors can be included and quality checked. Generally SQI and the data consistency algorithms (DCIC, DCPC) should always be applied independent of the weather situation, number, and type of receiver. The idea is that with the data consistency check and $F_{\text {frac }}$, irregularities like airplanes or birds might be detected and can be dismissed.

For operational usage, weights and algorithm applications can be stored in a lookup table. Generally, for a more precise analysis of the weight values for a specific meteorological situation, the horizontal wind fields derived by monostatic or bistatic multiple-Doppler radar measurements can be compared with each other, and the weight's choice can be analyzed. Furthermore, the dynamical parameters of mesoscale numerical simulations can be used as a reference field in order to analyze the impact and range of each weight.

The comprehensiveness and the emphasis on the quality-control scheme varied according to the weather situations, as demonstrated in this paper. During the stratiform precipitation event, for instance, the quality of horizontal wind is dominated by $F_{\text {stdev }}$, which is fixed for an experimental setup. The low impact is observed by $F_{\nabla Z}$ and $F_{\text {frac }}$. The cases discussed here show clearly that quality-control algorithms must be flexibly and independently applicable. The quality-control algorithm can also be applied to other horizontal wind field measurements, for example, monostatic dual-Doppler winds, or to measurements of radial wind components like ground-based or airborne Doppler lidar, radar, or sodar systems. The idea to create an averaged qualitycontrol field is applicable for all kinds of measurements within the three-dimensional space. Furthermore, if radar data are manipulated, for instance, clutter removal, correction for anomalous propagation, dealiasing, or fall speed correction, they can be flagged according to principles of the averaged quality-control field in order to give information about the comprehensiveness of the manipulation.

Bistatic receivers have to be arranged around a monostatic Doppler radar in a way to achieve high accuracy of wind measurements, large spatial coverage, high spatial resolution, a low minimum detectable reflectivity factor, measurements close to the ground, and a high accuracy of the horizontal wind field determination. The arrangement is determined by the distance between the transmitter and receiver and the antenna's look angle. As proposed in section $4 \mathrm{c}(1)$, the area with $\beta_{b}$ ranging between $25^{\circ}$ and $70^{\circ}$ provides measurements with the standard deviation of the horizontal wind field ranging between 2 and $3 \mathrm{~m} \mathrm{~s}^{-1}$, and a resolution ranging between 180 and $1200 \mathrm{~m}$ (for a pulse length $c \tau=300 \mathrm{~m}$ ). As a result, the bistatic antenna should be positioned to observe this area. As shown in Fig. 9, the observation area can be extended by setting the lower limit to $\beta_{b}=$ $20^{\circ}$ or $15^{\circ}$. On the other hand, the bistatic antenna's view angle should not be rotated closer to the baseline. Low minimum detectable reflectivity factors can be achieved when transmitting and receiving with vertical polarization. A comparison in terms of optimal arrangement of bistatic and monostatic Doppler radar networks is given in de Elia and Zawadzki (2001) and Friedrich and Hagen (2003, manuscript submitted to Meteor. Appl., hereinafter FAH03). In order to achieve measurements with $\sigma_{\left|\mathbf{v}_{h}\right|} \leq 3 \mathrm{~m} \mathrm{~s}^{-1}$ and a resolution of about $300 \mathrm{~m}$, the distance between transmitter and receiver should range between 40 and $50 \mathrm{~km}$ (FAH03).

Furthermore, scatterplots like in Figs. 4 and 7 allow for assessing the measurement error because the variance measured by a radar can be caused by a number of factors, for example, wind shear and turbulence. It is shown that the measurement error within the turbulent weather situation, for example, a convective situation, is much higher (85\% lie within $1 \mathrm{~m} \mathrm{~s}^{-1}$ ) than in stratiform precipitation (97\%-99\% of the Doppler velocities are within $1 \mathrm{~m} \mathrm{~s}^{-1}$ ).

Acknowledgments. We thank Hermann Scheffold, Hans Krafcyk, and Fred Ritenberg for their technical support at both radar systems. Furthermore, we thank the staff at DLR Weilheim and the Klaus Hager military weather service at Lagerlechfeld for their great support during the installation and operation of the bistatic network. We also thank Jörg Seltman (German Weather Service, Hohenpeissenberg) for data acquisition. Many thanks go to the radar team at McGill University, Montreal, Canada, especially Isztar Zawadzki and Alain Caya, for introduction to the retrieval program, as well as to Ramon de Elia for productive discussions and incentives. Furthermore, we say thank you to BINET Coop. (Josh Wurman, Mitch Randall, and Chris Burghard) for all their support during the installation and operation process. We have also benefited from constructive comments and changes suggested by the anonymous reviewers. Thanks are also given to Nerissa Röhrs, who helped us with the English language.

\section{REFERENCES}

Alberoni, P. P., and Coauthors, 2002: Quality and assimilation of radar data for NWP-A review. COST-717 working document EUR 20600, 38 pp. [Available online at http://www.smhi.se/cost717/.]

de Elia, R. and I. Zawadzki, 2000: Sidelobe contamination in bistatic radars. J. Atmos. Oceanic Technol., 17, 1313-1329.

- , and -2001 : Optimal layout of a bistatic radar network. $J$. Atmos. Oceanic Technol., 18, 1184-1194.

Doviak, R. J., P. S. Ray, R. G. Strauch, and L. J. Miller, 1976: Error estimation in wind fields derived from dual-Doppler radar measurement. J. Appl. Meteor., 15, 868-878.

Einfalt, T., B. Maul-Koetter, and S. Spies, 2000: A radar data quality control scheme used in hydrology. Phys. Chem. Earth, 25B, 1141-1146.

Friedrich, K., 2002: Determination of three-dimensional wind-vector fields using a bistatic Doppler radar network. Ph.D. thesis, Fak- 
ultaet fuer Physik, Ludwig-Maximilians-Universitaet Muenchen, 135 pp. [Available online at http://www.op.dlr.de/ pa4k/.]

— by a bistatic radar network during a downburst-producing thunderstorm. J. Atmos. Oceanic Technol., in press.

—- M. Hagen, and P. Meischner, 2000: Vector wind field determination by bistatic multiple-Doppler radar. Phys. Chem. Earth 25B, 1205-1208.

Hagen, M., 1997: Identification of ground clutter by polarimetric radar. Preprints, 28th Conf. on Radar Meteorology, Austin, TX, Amer. Meteor. Soc., 67-68.

James, C. N., and R. A. Houze, 2001: A real-time four-dimensional Doppler dealiasing scheme. J. Atmos. Oceanic Technol., 18, 1674-1683.

_ S. R. Brodzik, H. Edmon, R. A. Houze, and S. E. Yutter, 2000: Radar data processing and visualization over complex terrain. Wea. Forecasting, 15, 327-338.

Laroche, S., and I. Zawadzki, 1994: A variational analysis method for retrieval of three-dimensional wind field from single-Doppler radar data. J. Atmos. Sci., 51, 2664-2682.

$\ldots$, and — 1995: Retrievals of horizontal winds from singleDoppler clear-air data by methods of cross correlation and variational analysis. J. Atmos. Oceanic Technol., 12, 721-738.

Lee, R., G. D. Bruna, and J. Joss, 1995: Intensity of ground clutter and of echoes of anomalous propagation and its elimination. Preprints, 27th Conf. on Radar Meteorology, Vail, CO, Amer. Meteor. Soc., 651-652.

Mohr, C. G., L. Miller, R. Vaughan, and H. Frank, 1986: The merger of mesoscale datasets into a common Cartesian format for efficient and systematic analyses. J. Atmos. Oceanic Technol., 3, 143-161.

Montmerle, T., A. Caya, and I. Zawadzki, 2001: Simulation of a midlatitude convective storm initialized with bistatic Doppler radar data. Mon. Wea. Rev., 129, 1949-1967.

Nissen, R., D. Hudak, S. Laroche, R. de Elia, I. Zawadzki, and Y. Asuma, 2001: 3D wind field retrieval applied to snow events using Doppler radar. J. Atmos. Oceanic Technol., 18, 348-362.

Protat, A., and I. Zawadzki, 1999: A variational method for real-time retrieval of three-dimensional wind field from multiple-Doppler bistatic radar network data. J. Atmos. Oceanic Technol., 16, 432449.

Ray, S. P., K. K. Wagner, K. W. Johnson, J. J. Stephens, W. C. Bumgarner, and E. A. Mueller, 1978: Triple-Doppler observations of a convective storm. J. Appl. Meteor., 17, 1201-1212.

Satoh, S., and J. Wurman, 1999: Accuracy of composite wind fields derived from a bistatic multiple-Doppler radar network. Preprints, 29th Conf. on Radar Meteorology, Montreal, QC, Canada, Amer. Meteor. Soc., 221-224.

Schroth, A. C., M. S. Chandra, and P. Meischner, 1988: A C-band coherent polarimetric radar for precipitation and cloud physics research. J. Atmos. Oceanic Technol., 5, 803-822.

Seltmann, E. E. J., 2000: Clutter versus radar winds. Phys. Chem. Earth, 25B, 1173-1178.

Skolnik, M., 1990: Radar Handbook. McGraw-Hill, 1200 pp.

Takaya, Y., and M. Nakazato, 2002: Error estimation of the synthesized two-dimensional horizontal velocity in a bistatic Doppler radar system. J. Atmos. Oceanic Technol., 19, 74-79.

Testud, J., and M. Chong, 1983: Three-dimensional wind field analysis from dual-Doppler radar data. Part I: Filtering, interpolation and differentiating the raw data. J. Climate Appl. Meteor., 22, $1204-1215$.

Wurman, J., S. Heckman, and D. Boccippio, 1993: A bistatic multipleDoppler radar network. J. Appl. Meteor., 32, 1802-1814.

Yamada, Y., and M. Chong, 1999: VAD-based determination of the Nyquist interval number of Doppler velocity aliasing without wind information. J. Meteor. Soc. Japan, 77, 447-457. 